\title{
Performance evaluation of rough thrust pad bearing under thermo-elastohydrodynamic lubrication using an improved iterative method
}

\author{
Rahul Kumar ${ }^{1}$, Mohammad Sikandar Azam ${ }^{1}$, Subrata Kumar Ghosh ${ }^{1,}$, and Hasim Khan ${ }^{2}$ \\ ${ }^{1}$ Department of Mechanical Engineering, Indian Institute of Technology (ISM), Dhanbad, India \\ ${ }^{2}$ Department of Mathematics, College of Sciences, Jazan University, Jazan, Saudi Arabia
}

Received: 14 March 2018 / Accepted: 29 June 2018

\begin{abstract}
The asperities present on interacting surfaces of a bearing influence the film formation when the oil film becomes thinner and thinner. The aim of this article is to study the effect of stochastic roughness on bearing performance under thermo-piezoviscous and elastic condition using an average flow model. To investigate the present operating conditions, progressive mesh densification method as a fast and simple algorithm has been applied. The results obtained indicate that transverse roughness generates higher pressure compared to other orientational roughness at various film thicknesses. Maximum pressure, mass flow rate and load capacity are larger in transverse roughness compared to other orientational roughness for all values of hydrodynamic roughness parameters. A large sensitivity in load capacity for transverse orientation compared to longitudinal at higher film thickness and small film thickness ratios are witnessed. The frictional coefficient in longitudinal orientation is large compared to transverse orientation at all values of film thickness ratio and step ratios. Materials with low elastic modulus undergo large deformation, resulting in generation of two sharp pressure peaks. These results may possess good acceptability to practical applications for studying the effect of surface roughness under thermo-elastohydrodynamic lubrication condition.
\end{abstract}

Keywords: Stochastic roughness / thermo-elastohydrodynamic lubrication / transverse roughness / longitudinal roughness

\section{Introduction}

More attention has been given to lubricant's flow under thin film lubrication in recent times. For large operating efficiency, lubricants with low viscosity are used under high load and high velocity. Under this lubrication regime, operating conditions become much more severe when surface roughness or asperities present on interacting surfaces influence the film formation. The effect of these asperities on performance parameters of the bearing cannot be ignored with reduced film thickness.

Compared to counterformal contacts, the film formation is more in conformal contacts that appear in thrust bearings, journal bearings, and mechanical seals. Previous studies suggest that at high load and high sliding velocity, surface deformation and piezoviscous effect appear under hydrodynamic lubrication. The theory of hydrodynamic lubrication is believed to be valid under small load and small sliding velocity. With decrease in film thickness, the

\footnotetext{
* e-mail: subratarec@yahoo.co.in
}

deflection of bounding surfaces becomes comparable to film thicknesses that are caused due to high generated pressure. Carl [1] investigated that small surface deformation caused by a nominal pressure of $7 \mathrm{MPa}$ or less cannot be neglected. Hemingway [2] suggested that the surface deformation under small pressure of $2 \mathrm{MPa}$ plays a significant role in film formation. Kawabata et al. [3] analyzed the elastohydrodynamic lubrication condition in thrust pad bearing with circular bumps and different inlet conditions. Many researches [4-7] gave more importance to thermal expansion compared to elastic deformation in conformal contacts. Cameron [8] studied the effect of generated heat on viscosity of the lubricant under conformal contacts. The combined effect of elastic deformation and thermopiezoviscous effect on lubricant in conformal contact has been given less importance till date compared to counterformal contact.

Lundberg [9] investigated that microscopic scale roughness gets imparted on engineering surfaces during finishing operations like grit blasting, lapping, and grinding. Lubrication is affected by surface roughness in various ways. Bakolas [10] suggested that microscopic 


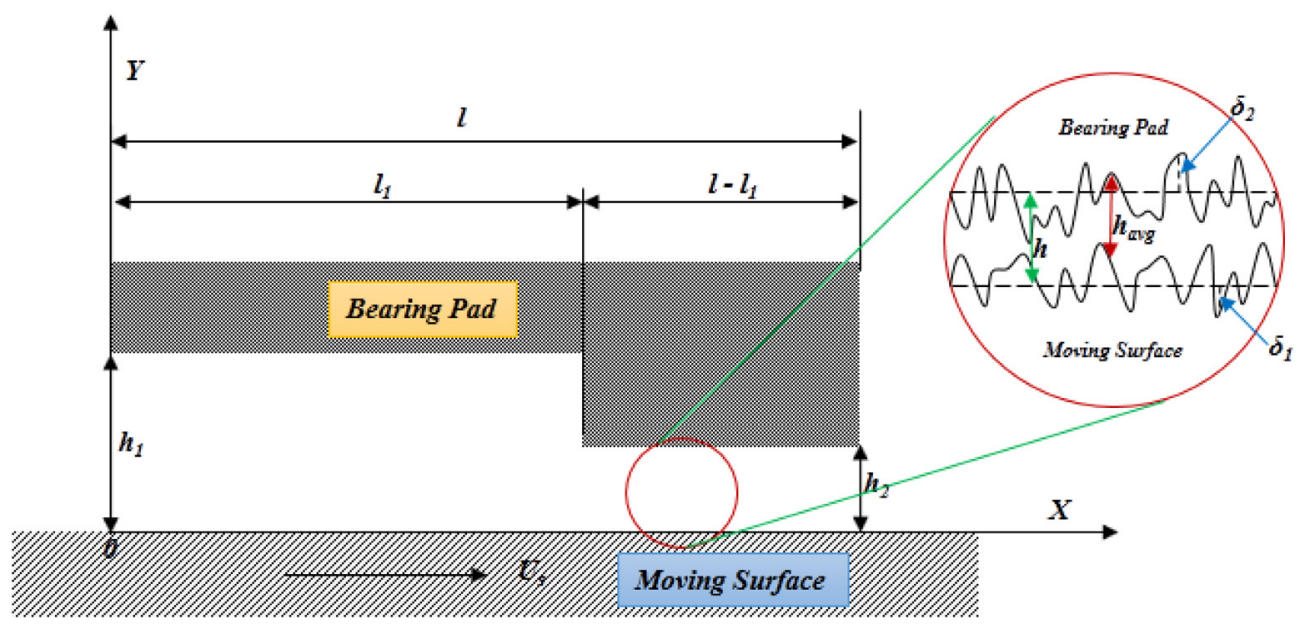

Fig. 1. Illustrative sketch of Rayleigh step bearing with stochastic roughness.

roughness disturbs the inlet pressure built up along with coefficient of friction and stress field at subsurface. Two different models were utilized to evaluate the effect of roughness on lubricant film. The first model was based on stochastic method. Tzeng and Sabiel [11] introduced this model by treating the surface roughness as a random variable having characteristics of a probability distribution function. Based on stochastic model, high amount of work was done on one-dimensional roughness pattern [12-16]. An average flow model was derived by Patir \& Cheng [17-19], where coefficients called flow factors were used to modify the Reynolds equation to study the effect of two-dimensional roughness. This average model was utilized to study the roughness effect on average film thickness and pressure distribution. This model was called as PC model for average flow and is widely accepted till date [20-26]. These works utilized the flow factor formula suggested by Patir and Cheng, which used isotropic Gaussian surface and neglected the effect of roughness directional pattern, kurtosis, and skewness. Qiu and Raeymaekers [27] studied the effect of stochastic roughness present inside textured bearing surface on performance parameters of a parallel slider bearing. Wang et al. [28] utilized the PC model to study the effect of roughness parameters along with roughness direction, skewness, and kurtosis on performance parameters of a thrust pad bearing lubricated with water.

The second model was based on deterministic approach in which roughness values are directly implemented in film thickness equation. The film thickness and pressure distribution are simulated using this deterministic model to study its effect on performance parameters of the bearing. This deterministic model was implemented in lubrication problem having a well-defined geometry [29-36].

All these studies suggest that the effect of elastic deformation, thermo-piezoviscous effect of lubricant, and surface roughness (stochastic) in conformal contacts has been given less importance till date compared to counterformal contacts under thin film lubrication. Although, these conditions affect the performance parameters of a bearing, no literature reports the combined effect of all these conditions on performance parameters under thin film lubrication.
The present article deals with the thermo-elastohydrodynamic simulation of a one-dimensional Rayleigh step bearing with rough (stochastic) interacting surfaces using an improved method of direct iteration. The effect of directional orientation of surface roughness and hydrodynamic roughness parameter on load capacity, frictional coefficient, total mass flow rate, and maximum temperature has been studied. The results obtained under different orientations have been compared with those obtained under smooth conditions to showcase a detailed view of roughness effect on bearing performance.

\section{Mathematical modeling}

Figure 1 represents the illustrative sketch of a onedimensional Rayleigh step bearing with stochastic roughness on both the surfaces with same directional pattern $\left(\gamma_{1}=\gamma_{2}=\gamma\right)$ and same standard deviation of roughness heights $\left(\sigma_{1}=\sigma_{2}\right)$ with combined standard deviation $\left(\sigma=\sqrt{\sigma_{1}^{2}+\sigma_{2}^{2}}\right)$. The contact area consists of a stationary stepped pad of width $l$ and a flat runner moving with velocity $U_{s}$. The directional advancement of runner is from left to right.

\subsection{Governing equations}

The modified form of one-dimensional average Reynolds equation considering stochastic roughness $[37,38]$ to calculate hydrodynamic pressure is given as follows:

$$
\frac{\partial}{\partial x}\left(\phi_{x} \frac{\rho h^{3}}{12 \eta} \frac{\partial p}{\partial x}\right)-\frac{U_{s}}{2} \frac{\partial\left(\rho h_{a v g}\right)}{\partial x}=0,
$$

where $h$ is the nominal film thickness, $h_{\text {avg }}$ is the average film gap between two surfaces, $\eta$ is the lubricant's viscosity, $\rho$ is the lubricant's density, $p$ is the generated pressure in the lubricant film, $\phi_{x}$ is the pressure flow factor, and $U_{s}$ is the moving surface's sliding velocity.

The average film gap between two surfaces is given by

$$
h_{\mathrm{avg}}=\int_{-h}^{\infty}(h+\delta) f(\delta) d \delta,
$$


where normal probability density function $f(\delta)$ for Gaussian distribution is defined as

$$
f(\delta)=\frac{1}{\sigma \sqrt{2 \pi}} \exp \left(-\frac{\delta^{2}}{2 \sigma^{2}}\right) .
$$

By introducing the dimensionless parameters in equation (1), the dimensionless form of modified average Reynolds equation is as follows:

$$
\frac{\partial}{\partial X}\left(\varphi_{x} \frac{\rho^{*} H^{3}}{\eta^{*}} \frac{\partial P}{\partial X}\right)-\frac{\partial\left(\rho^{*} H_{\mathrm{avg}}\right)}{\partial X}=0
$$

where $X={ }^{x} / l, P=p h_{2}{ }^{2} / 6 U s l \eta_{0}, H={ }^{h} / h_{2}, \rho^{*}=\rho / \rho_{0}$, $\eta^{*}=\eta / \eta_{0}, H_{\text {avg }}=h_{\text {avg }} / h_{2}$.

The discretized form (using FDM) of the dimensionless equation (4) is given by

$$
P_{i}=\frac{1}{A+B}\left(\left(A \times P_{i+1}\right)+\left(B \times P_{i-1}\right)-\Delta X(C-D)\right),
$$

where

$$
\begin{gathered}
A=\frac{0.5\left(\phi_{x_{i}}+\phi_{x_{i+1}}\right) \times 0.5\left(\rho_{i}^{*}+\rho_{i+1}^{*}\right) \times\left[0.5\left(H_{i}+H_{i+1}\right)\right]^{3}}{0.5\left(\eta_{i}^{*}+\eta_{i+1}^{*}\right)}, \\
B=\frac{0.5\left(\phi_{x_{i}}+\phi_{x_{i-1}}\right) \times 0.5\left(\rho_{i}^{*}+\rho_{i-1}^{*}\right) \times\left[0.5\left(H_{i}+H_{i-1}\right)\right]^{3}}{0.5\left(\eta_{i}^{*}+\eta_{i-1}^{*}\right)}, \\
C=0.5\left(\rho_{i}^{*}+\rho_{i+1}^{*}\right) \times 0.5\left(H_{\mathrm{avg}_{i}}+H_{\mathrm{avg}_{i+1}}\right), \\
D=0.5\left(\rho_{i}^{*}+\rho_{i-1}^{*}\right) \times 0.5\left(H_{\mathrm{avg}_{i}}+H_{\mathrm{avg}_{i-1}}\right) .
\end{gathered}
$$

The boundary conditions satisfying equation (5) are $P=0$ for $X=0$ and $X=1$.

\subsection{Film thickness equation}

The film thickness equation considering elastic deformation of the interacting surfaces (assuming them semiinfinite bodies) is given by

$$
h=\left\{\begin{array}{l}
h_{1}+d, \text { for } \quad 0 \leq x<l_{1} \\
h_{2}+d, \text { for } \quad l_{1} \leq x \leq l,
\end{array}\right.
$$

where $d$ represents the equivalent elastic deformation of the interacting surfaces.

The equivalent elastic deformation of the interacting surfaces at any point $x$ due to pressure at point $s$ is governed by the following equation:

$$
\begin{gathered}
d=-\frac{4}{\pi E^{\prime}} \int_{0}^{l} p(s) \log |x-s| d s \\
\frac{2}{E^{\prime}}=\frac{\left(1-\vartheta_{1}^{2}\right)}{E_{1}}+\frac{\left(1-\vartheta_{2}^{2}\right)}{E_{2}},
\end{gathered}
$$

where $E^{\prime}$ is the equivalent elastic modulus, $E_{1}$ is the Young's moduli of bearing pad, and $E_{2}$ is the Young's moduli of moving surface. $\vartheta_{1}$ and $\vartheta_{2}$ are the Poission's ratios of the respective materials.

The dimensionless form of the equations (6) and (7) are

$$
H= \begin{cases}k+d^{*}, \text { for } & 0 \leq X<L_{1} \\ 1+d^{*}, \text { for } & L_{1} \leq X \leq 1,\end{cases}
$$

$$
\begin{aligned}
d^{*} & =\left(\frac{-24 \eta_{0} U_{s} l^{2}}{\pi E^{\prime} h_{2}^{3}}\right) \int_{0}^{1} P(S) \log |X-S| d S \\
& =-\psi \int_{0}^{1} P(S) \log |X-S| d S,
\end{aligned}
$$

where $\psi=\left(\frac{24 \eta_{0} U_{s} l^{2}}{\pi E^{\prime} h_{2}{ }^{3}}\right)$ and $k=\frac{h_{1}}{h_{2}}$ is the film thickness
ratio.

The discretized form of equation (9) can be written as

$$
H_{i}= \begin{cases}k-\psi \sum_{i=1}^{N} D_{i j} P_{j}, \text { for } & 0 \leq X<L_{1} \\ 1-\psi \sum_{i=1}^{N} D_{i j} P_{j}, \text { for } & L_{1} \leq X \leq 1,\end{cases}
$$

where

$$
\begin{aligned}
D_{i j}= & \left(i-j+\frac{1}{2}\right) \Delta\left(\ln \left(\left|i-j+\frac{1}{2}\right| \Delta\right)-1\right) \\
& -\left(i-j-\frac{1}{2}\right) \Delta\left(\ln \left(\left|i-j-\frac{1}{2}\right| \Delta\right)-1\right) .
\end{aligned}
$$

The discretized (dimensionless) form of average film gap is

$$
\begin{aligned}
H_{\mathrm{avgi}}= & \frac{H_{i}}{2}\left\{1+\operatorname{erf}\left(\frac{H_{i}}{\sqrt{2 \sigma}^{*}}\right)\right\} \\
& +\frac{\sigma^{*}}{\sqrt{2 \pi}} \exp \left(-\frac{H_{i}^{2}}{2 \sigma^{* 2}}\right),
\end{aligned}
$$
where $\sigma^{*}=\sigma / h_{2}$ and $\operatorname{erf}\left(\frac{H_{i}}{\sqrt{2} \sigma}\right)$ is the error function and is
defined as

$$
\operatorname{erf}\left(\frac{H_{i}}{\sqrt{2} \sigma^{*}}\right)=\frac{2}{\sqrt{\pi}} \int_{0}^{\frac{H_{i}}{\sqrt{2} \sigma^{*}}} \exp \left(-t^{2}\right) d t .
$$

The discretized (dimensionless) form of pressure flow factor [17] is

$$
\phi_{x i}=\left\{\begin{array}{l}
1-c e^{-r\left(\frac{H_{i}}{\sigma^{*}}\right)}, \text { for } \gamma \leq 1, \\
1+c\left(\frac{H_{i}}{\sigma^{*}}\right)^{-r}, \text { for } \gamma>1 .
\end{array}\right.
$$




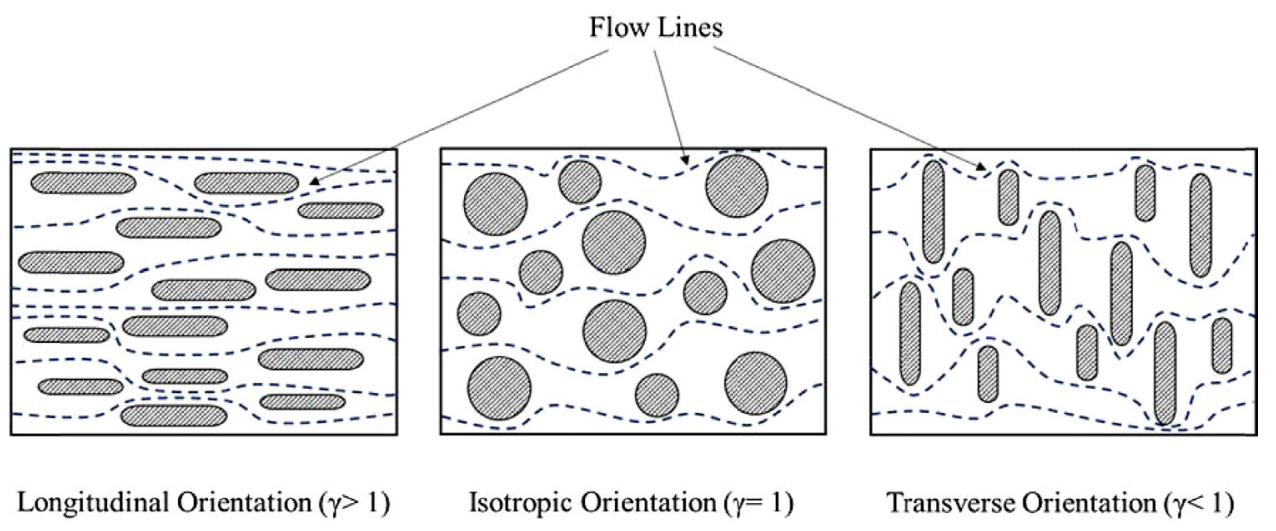

Fig. 2. Illustrative sketch of roughness orientation in the contact area.

Table 1. Constants for equation (14).

\begin{tabular}{lllll}
\hline$\gamma$ & $c$ & $r$ & Range & Roughness orientation \\
\hline $1 / 9$ & 1.480 & 0.42 & $H / \sigma^{*}>1.00$ & \\
$1 / 6$ & 1.380 & 0.42 & $H / \sigma^{*}>1.00$ & Transverse roughness \\
$1 / 3$ & 1.180 & 0.42 & $H / \sigma^{*}>0.75$ & Isotropic roughness \\
1 & 0.900 & 0.56 & $H / \sigma^{*}>0.50$ & \\
3 & 0.225 & 1.50 & $H / \sigma^{*}>0.50$ & Longitudinal roughness \\
6 & 0.520 & 1.50 & $H / \sigma^{*}>0.50$ & \\
9 & 0.870 & 1.50 & & \\
\hline
\end{tabular}

Source: Adapted from reference [17].

The constants $r$ and $c$ are function of roughness pattern $\gamma$ (as shown in Fig. 2) and have been defined in Table 1. The pressure flow factor $\left(\varphi_{x}\right)$ is the correction factor for pressure-induced flow (Poiseuille flow) in rough condition. It compares the pressure-induced flow in smooth surface with rough surface. Its dependency on hydrodynamic roughness parameter $\left(\Lambda=H / \sigma^{*}\right)$ is shown in Figure 3 .

\subsection{Lubricant's density and viscosity equations}

The discretized form of dimensionless density-pressuretemperature relationship with linear thermal correction factor [39] used in the present study is expressed as

$$
\begin{aligned}
\rho_{i}{ }^{*}= & {\left[1+\frac{0.6 \times 10^{-9} \times\left(\frac{6 \eta_{0} U_{s} l}{h_{2}^{2}}\right) P_{i}}{1+1.7 \times 10^{-9} \times\left(\frac{6 \eta_{0} U_{s} l}{h_{2}{ }^{2}}\right) P_{i}}\right] } \\
& \times\left(1-\beta T_{0}\left(T_{i}{ }^{*}-1\right)\right),
\end{aligned}
$$

where $T_{0}$ is the lubricant's temperature at inlet, $\beta$ is the thermal expansivity coefficient of the lubricant, and $T_{i}^{*}$ is the dimensionless temperature in lubricant film at node $i$.

The discretized form of dimensionless viscosity-temperature-pressure relationship [40] used in the present study is expressed as

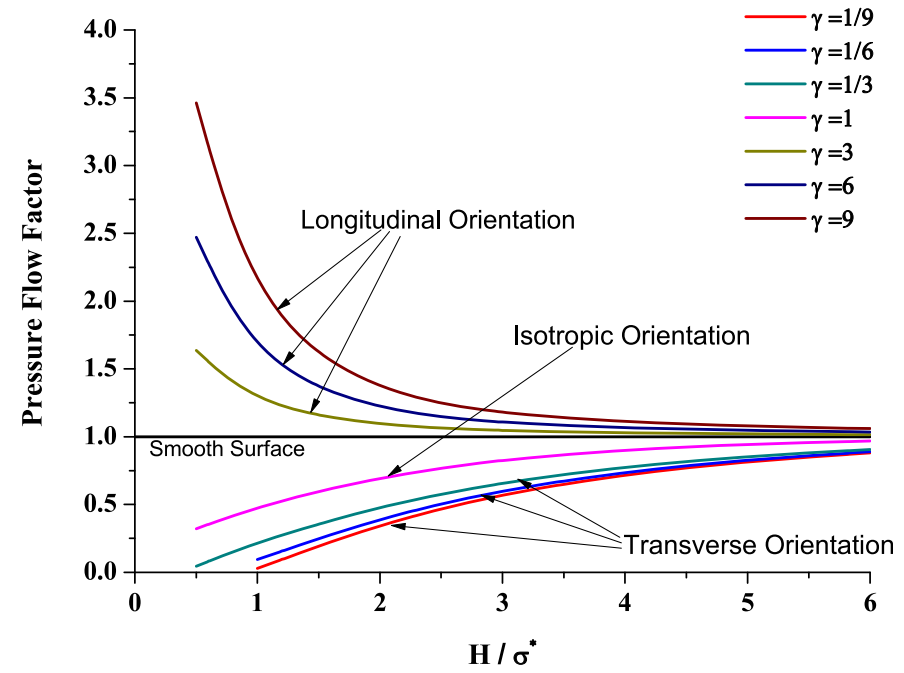

Fig. 3. Dependency of pressure flow factor on hydrodynamic roughness parameter (adapted from Ref. [17]).

$$
\begin{aligned}
\eta_{i}{ }^{*}= & \exp \left\{\left[\left\{l n \eta_{0}+9.67\right\} \times\left\{-1+\left(1+5.1 \times 10^{-9}\right.\right.\right.\right. \\
& \left.\left.\left.\left.\left.\times\left(\frac{6 \eta_{0} U_{s} l}{h_{2}{ }^{2}}\right) P_{i}\right)^{Z}\right\}\right]-\zeta T_{0}\left(T_{i}{ }^{*}-1\right)\right)\right\}
\end{aligned}
$$




$$
Z=\frac{\alpha}{\left(\ln \eta_{0}+9.67\right)\left(5.1 \times 10^{-9}\right)},
$$

where $\zeta$ and $\alpha$ are thermal viscosity coefficient and pressure viscosity coefficient of the lubricant, respectively.

\subsection{Energy equation}

Energy equation for one-dimensional Rayleigh step bearing with stochastic roughness is given by

$$
\begin{array}{r}
\rho C_{p}\left(U_{s} \frac{\partial T}{\partial x}+W \frac{\partial T}{\partial z}\right)=k\left(\frac{\partial^{2} T}{\partial x^{2}}+\frac{\partial^{2} T}{\partial z^{2}}\right) \\
-\frac{T}{\rho} \frac{\partial \rho}{\partial T}\left(U_{s} \frac{\partial p}{\partial x}+W \frac{\partial p}{\partial z}\right)+\eta\left(\frac{\partial U_{s}}{\partial z}\right)^{2} .
\end{array}
$$

Integrating the energy equation (18) across the average film thickness by assuming that (a) $\eta, T$, and $p$ do not change across the film thickness, (b) convective heat transfer across film thickness, conductive heat along film, and effect of temperature on $\rho$ is negligible [41], the one-dimensional energy equation for temperature distribution is

$$
q_{x} \frac{\partial T}{\partial x}=\frac{\eta U_{s}^{2}}{\rho C_{p} h_{\mathrm{avg}}}+\frac{h_{\mathrm{avg}}^{3}}{12 \rho C_{p} \eta}\left(\frac{\partial p}{\partial x}\right)^{2},
$$

where $q_{x}=\frac{U_{s} h_{\mathrm{avg}}}{2}-\phi_{x} \frac{h^{3}}{12 \eta} \frac{\partial p}{\partial x}$ is the flow rate per width and $C_{p}$ is the lubricant's specific heat.

Using the dimensionless parameter, $Q_{x}=\frac{q_{x}}{U_{s} h_{2}}$.

The dimensionless form of temperature distribution equation (19) is

$$
\frac{\partial T^{*}}{\partial X}=\frac{1}{\varphi Q_{x} \rho^{*}}\left\{\frac{\eta^{*}}{H_{\mathrm{avg}}}+\frac{3 H_{\mathrm{avg}}}{\eta^{*}}\left(\frac{\partial P}{\partial X}\right)^{2}\right\}
$$

where $Q_{x}=\frac{H_{\text {avg }}}{2}-\phi_{x} \frac{H^{3}}{2 \eta^{*}} \frac{\partial P}{\partial X}$ and $\varphi=\frac{T_{0} h_{2}{ }^{2} \rho_{0} C_{p}}{\eta_{0} U_{s} l}$.

The discretized form of dimensionless energy equation (20) is

$$
\frac{T_{i}^{*}-T_{i-1}^{*}}{\Delta X}=\frac{1}{\varphi Q_{x i} \rho_{i}^{*}}\left\{\frac{\eta_{i}{ }^{*}}{H_{\mathrm{avg}_{i}}}+\frac{3 H_{\mathrm{avg}_{i}}}{\eta_{i}{ }^{*}}\left(\frac{P_{i}-P_{i-1}}{\Delta X}\right)^{2}\right\},
$$

where $Q_{x i}=\frac{H_{\mathrm{avg}_{i}}}{2}-\phi_{x i} \frac{H_{i}{ }^{3}}{\eta_{i}^{*}} \frac{P_{i}-P_{i-1}}{\Delta X}$.

\subsection{Mass flow rate}

Total mass flow rate per unit width of the lubricant is

$$
\begin{gathered}
q=q_{c}+q_{p} \\
q_{c}=\frac{\rho U_{s} h_{\mathrm{avg}}}{2}, q_{p}=-\phi_{x} \frac{\rho h^{3}}{12 \eta} \frac{\partial p}{\partial x},
\end{gathered}
$$

where, $q_{c}, q_{p}$, and $q$ are the Couette, Poiseuille, and total mass flow rate, respectively.

Using the dimensionless parameters, the dimensionless form total mass flow rate equation (22) is

$$
Q=Q_{c}+Q_{p},
$$

where $Q=\frac{q}{\rho_{0} U_{s} h_{2}}, Q_{c}=\frac{q_{c}}{\rho_{0} U_{s} h_{2}}$, and $Q_{p}=\frac{q_{p}}{\rho_{0} U_{s} h_{2}}$,

$$
Q=\frac{\rho^{*} H_{\mathrm{avg}}}{2}-\phi_{x} \frac{\rho^{*} H^{3}}{2 \eta^{*}} \frac{\partial P}{\partial X} .
$$

The discretized form of dimensionless mass flow rate equation (24) is

$$
Q_{i}=\frac{\rho_{i}^{*} H_{\operatorname{avg}_{i}}}{2}-\phi_{x i} \frac{\rho_{i}^{*} H_{i}^{3}}{2 \eta_{i}^{*}}\left(\frac{P_{i}-P_{i-1}}{\Delta X}\right) .
$$

\subsection{Load capacity}

Integrating the pressure distribution over the entire contact area, the bearing's load capacity per unit length is given by

$$
w=\int_{0}^{l} p d x
$$

The dimensionless form of load capacity is

$$
w^{*}=\int_{0}^{1} P d X
$$

where $w^{*}=\frac{w h_{2}{ }^{2}}{6 \eta_{0} U_{s} l^{2}}$ is the dimensionless parameter of the load capacity.

The discretized form of dimensionless load capacity equation (27) is

$$
w^{*}=\sum_{i=1}^{N} P_{i} \Delta X
$$

\subsection{Frictional coefficient}

Integrating the shear stress developed over the entire contact area, the frictional force on moving surface is given by

$$
f=\int_{0}^{l} \eta \frac{\partial U_{s}}{\partial z} \partial x=\int_{0}^{l}\left(-\eta \frac{U_{s}}{h_{\mathrm{avg}}}-\frac{h_{\mathrm{avg}}}{2} \frac{\partial p}{\partial x}\right) \partial x .
$$

Using the dimensionless parameter, the dimensionless form of friction equation (29) is

$$
F=\int_{0}^{1}\left(-\frac{\eta^{*}}{H_{\text {avg }}}-3 H_{\text {avg }} \frac{\partial P}{\partial X}\right) \partial X,
$$

where $F=\frac{f h_{2}}{\eta_{0} U_{s} l}$ is the dimensionless parameter. 


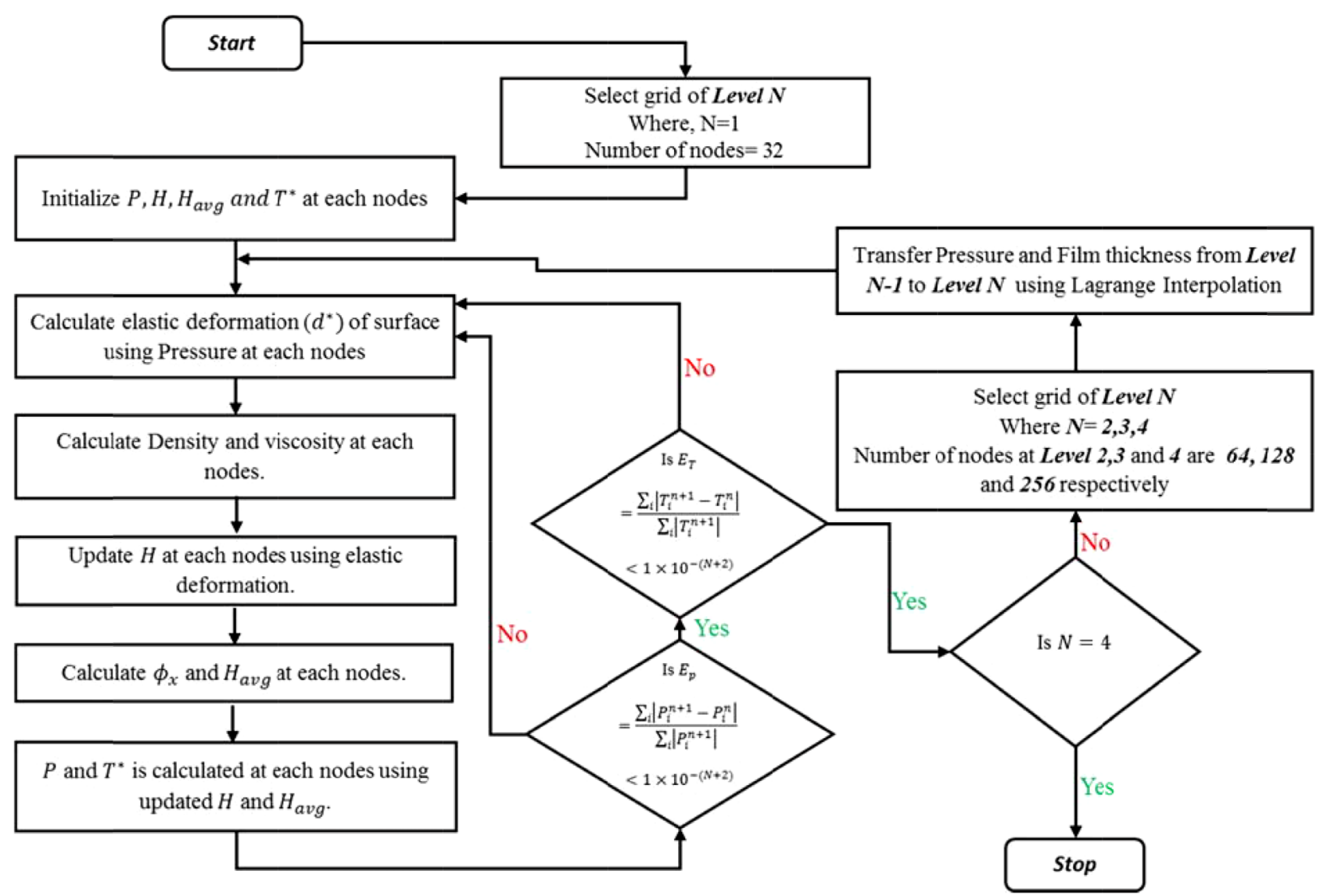

Fig. 4. Computational steps of PMD involved in the present study.

The dimensionless coefficient of friction is

$$
F^{*}=\frac{F}{w^{*}} .
$$

The above set of discretized dimensionless equations are solved iteratively using an improved method for direct iteration (progressive mesh densification (PMD) method) [42] for the present analysis. The steps involved in computation are in Figure 4. Pressure $P$ and temperature $T^{*}$ at inlet and outlet are set at zero and one respectively as the boundary conditions for temperature and pressure distribution.

\section{Results and discussion}

To study the effect of directional orientation of stochastic roughness on performance parameters of Rayleigh step bearing, the minimum film thickness $\left(h_{2}\right)$, film thickness ratios $(k)$, and step ratios $\left(L_{1}\right)$ are changed by keeping other parameters fixed. This is similar to changing of load by keeping other parameters fixed. For the present study, equivalent elastic modulus $(E=230.76 \mathrm{GPa})$ has been taken, which serves as a consolidation of two steel ( $E=210 \mathrm{GPa}, \vartheta=0.3$ ) bodies. The other essential parameters with fixed values are catalogued in Table 2.

\subsection{Validation of present algorithm}

Figure 5 compares the result obtained using present algorithm and that of Yagi and Sugimura [43] under elastohydrodynamic lubrication (EHL) condition. The results obtained using the present algorithm is in good agreement with the published one. This shows that present algorithm can serve as a simple and fast algorithm to study EHL condition. Using the present algorithm, effect of directional orientation on performance parameters of the Rayleigh step bearing has been studied under rough thermo-EHL condition in subsequent sections.

\subsection{Effect of directional orientation of roughness}

To study the effect of directional orientation of surface roughness on bearing performance parameters, a modified average Reynolds equation is derived on the basis of probabilistic properties and distribution of roughness height. A flow factor method was developed by Patir and Cheng [17] to study the effect of different directional orientations of surface roughness. Being a powerful method, it has wide acceptance till date in the field of thin film lubrication. This method uses a modified average Reynolds equation (1) implicating flow factor to inspect the response of fluid over the rough surface. The ratio of the minimum film thickness and standard deviation (SD) 
Table 2. Essential parameters and their values.

\begin{tabular}{lll}
\hline Basic parameters & Symbols & Values \\
\hline Width of the slider & $l$ & $10 \mathrm{~mm}$ \\
Difference in film thickness & $h_{1}-h_{2}$ & $100 \mathrm{~nm}$ \\
Equivalent elastic modulus & $E^{\prime}$ & $230.76 \mathrm{GPa}$ \\
Inlet viscosity of the lubricant & $\eta_{0}$ & $0.01 \mathrm{Pas}$ \\
Inlet density of the lubricant & $\rho_{0}$ & $846 \mathrm{~kg} / \mathrm{m}^{3}$ \\
Poisson's ratio of steel & $\vartheta$ & 0.30 \\
Sliding velocity & $U_{s}$ & $0.05 \mathrm{~m} / \mathrm{s}$ \\
Pressure-viscosity coefficient & $\alpha$ & $10 \mathrm{GPa}$ \\
Inlet temperature of the & $T_{0}$ & $313 \mathrm{~K}$ \\
lubricant & &
\end{tabular}

of roughness height (say hydrodynamic roughness parameter $(\Lambda)$ ) is used to express the flow factor (Eq. (14)). $\Lambda$ characterizes the surface irregularities. A higher value of $\Lambda$ represents smaller heights of asperities resulting into almost smoother surfaces. From average film gap and flow factor definition, $\phi_{x}$ and $H_{\text {avg }}$ tend to 1 and $H$, respectively, when $\sigma^{*}$ tends to 0 . In such a case, modified average Reynolds equation reduces to Reynolds equation of smooth surface. Generally, the values at $\Lambda \geq 6$ is equivalent to values obtained considering smooth surfaces. For the present analysis (thermo-piezoviscous and elastic condition), $\Lambda=3$ has been taken.

For different values of minimum film thickness $\left(h_{2}\right)$, the pressure generation is maximum $(0.75 \mathrm{MPa}$ at $1000 \mathrm{~nm}$ and $4 \mathrm{MPa}$ at $100 \mathrm{~nm}$ ) in case of bearing with transverse directional orientation of surface roughness (as shown in Fig. 6). Minimum pressure generation (0.42 MPa at $1000 \mathrm{~nm}$ and $3.85 \mathrm{MPa}$ at $100 \mathrm{~nm}$ ) is witnessed in the bearing with longitudinal orientation of roughness. The trend followed by pressure distribution under different directional orientation is as follows:

$$
p_{\text {transverse }}>p_{\text {isotropic }}>p_{\text {smooth }}>p_{\text {longitudnal }} .
$$

This trend depends on the pressure flow factor (also called correction factor) $\left(\phi_{x}\right)$ multiplied with the value of $\frac{\rho h^{3}}{\eta}$ of the Poiseuille flow term. For a particular value of $\Lambda$, the value of $\phi_{x}$ is maximum for longitudinal roughness and minimum for transverse roughness for different values of $\gamma$ (as shown in Fig. 3). Due to this, longitudinal roughness offers less resistance to pressure-induced flow, allowing only a very small amount of side flow. The pressureinduced flow is slightly larger in longitudinal roughness compared to smooth surface because the average gap in valleys are slightly larger than the nominal film thickness (as shown in Fig. 2). Under transverse roughness, the side flow increases and pressure-induced flow decreases, thus offering more resistance to the pressure-induced flow and results into larger pressure generation. For isotropic roughness, the side flow and pressure-induced flow are of same order. Due to this, the flow has to pass around the roughness orientation resulting into higher resistance to

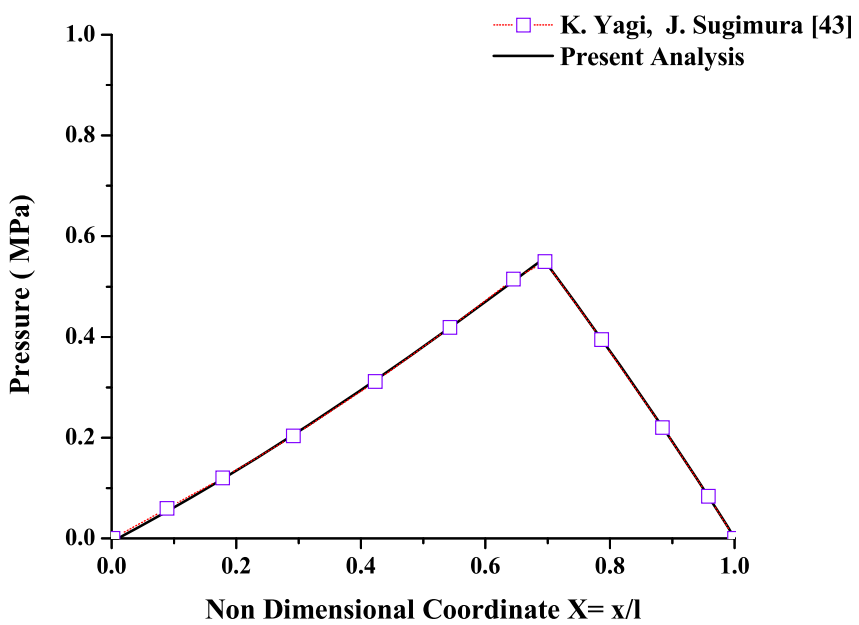

Fig. 5. Comparison between pressure distribution of present work and of Yagi and Sugimura [43] for EHL condition at $l=10 \mathrm{~mm}$, $L_{1}=0.7, E^{\prime}=462 \mathrm{GPa}, \eta_{0}=0.01 \mathrm{~Pa} \mathrm{~s}, \rho_{0}=846 \mathrm{Kg} / \mathrm{m}^{3}, \vartheta=0.30$, $U_{s}=0.05 \mathrm{~m} / \mathrm{s}, \alpha=10 \mathrm{GPa}^{-1}, h_{1}-h_{2}=100 \mathrm{~nm}$, and $h_{2}=1000 \mathrm{~nm}$.

the pressure-induced flow compared to smooth surfaces but lesser resistance to the pressure-induced flow compared to transverse roughness.

As $h_{2}$ decreases from 1000 to $100 \mathrm{~nm}$, the pressure generation in bearing increases for all orientations of roughness. But the difference between the maximum pressure of transverse and longitudinal roughness decreases from 0.3 to $0.15 \mathrm{MPa}$ with decrease in $h_{2}$ from 1000 to $100 \mathrm{~nm}$ (as shown in Fig. 6). Reason behind such type of change in maximum pressure is that the pressureinduced flow rate depends upon the film thickness and viscosity. In the value of $\phi_{x} \frac{\rho h^{3}}{\eta}$ of the pressure-induced flow term, film thickness raises to power equal to 3 . Due to reduction in $h$ and increase in viscosity due to pressure, the pressure-induced flow rate decreases, resulting into high pressure generation at smaller film thickness. At this high pressure, the surfaces deform and result into generation of a large no-pressure area at inlet at very small film thickness $(100 \mathrm{~nm})$. Asperities under different types of roughness orientations also deform under the influence of this high pressure and become almost close to smooth surface (as shown by film thickness distribution in Fig. 6). Due to asperity deformation, the difference between maximum pressure under different roughness orientations at very small film thickness $(100 \mathrm{~nm})$ becomes small compared to that at higher film thickness $(1000 \mathrm{~nm})$. This is because the difference between pressure-induced flows under different roughness orientations becomes very small at small film thickness $(100 \mathrm{~nm})$ (as shown in Fig. 8).

Temperature reduces the viscosity of the lubricant resulting into an increase in the pressure-induced flow rate. Thus, the pressure generated under thermo-piezoviscous and elastic condition for all roughness orientations is slightly lower compared to piezoviscous and elastic condition. 

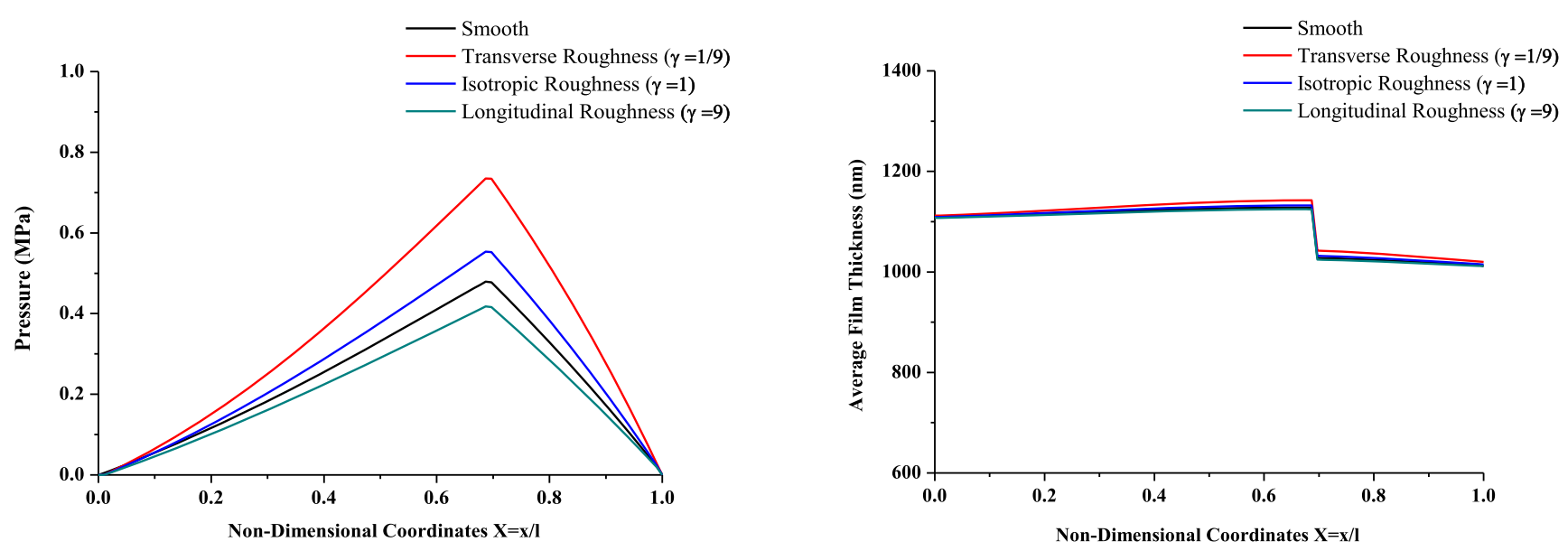

(a)
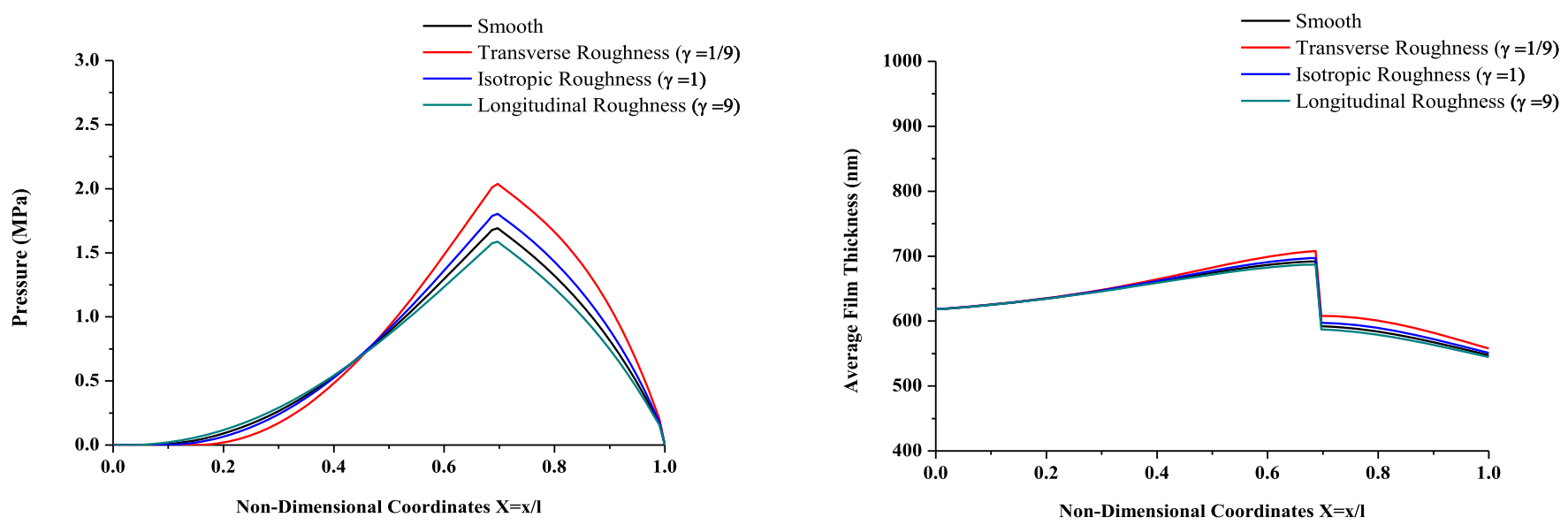

(b)
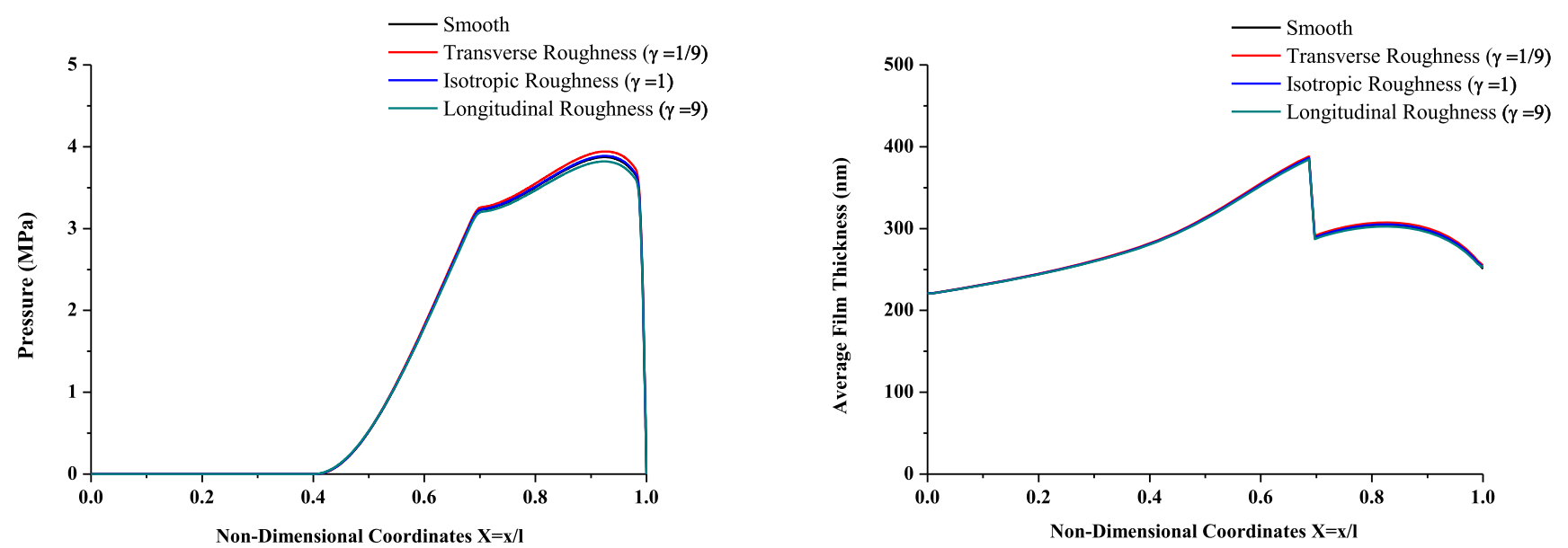

(c)

Fig. 6. Comparison of pressure and film thickness distribution for different directional orientation of surface roughness and smooth surface at $l=10 \mathrm{~mm}, \eta_{0}=0.01 \mathrm{~Pa} \mathrm{~s}, \rho_{0}=846 \mathrm{~kg} / \mathrm{m}^{3}, \vartheta=0.30, U_{s}=0.05 \mathrm{~m} / \mathrm{s}, \alpha=10 \mathrm{GPa}^{-1}, L_{1}=0.7, h_{1}-h_{2}=100 \mathrm{~nm}$, and $\Lambda=3$. (a) $h_{2}=1000 \mathrm{~nm}$, (b) $h_{2}=500 \mathrm{~nm}$, and (c) $h_{2}=100 \mathrm{~nm}$. 


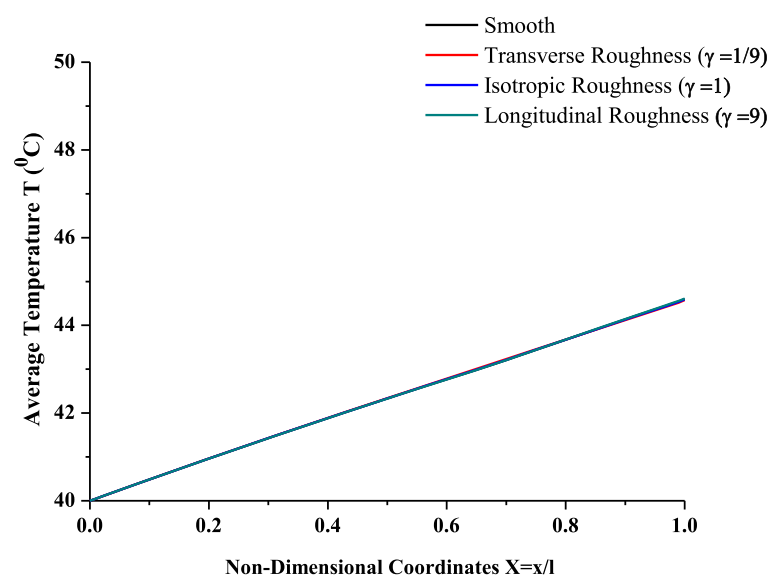

(a)

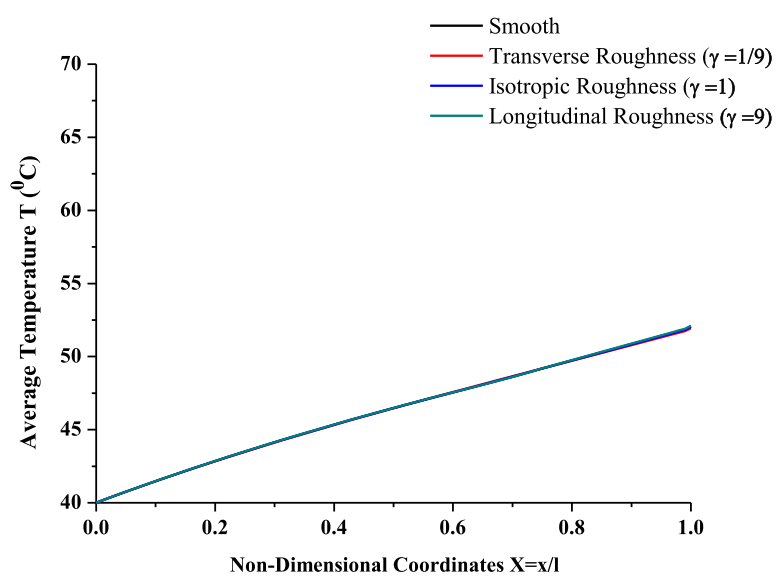

(b)

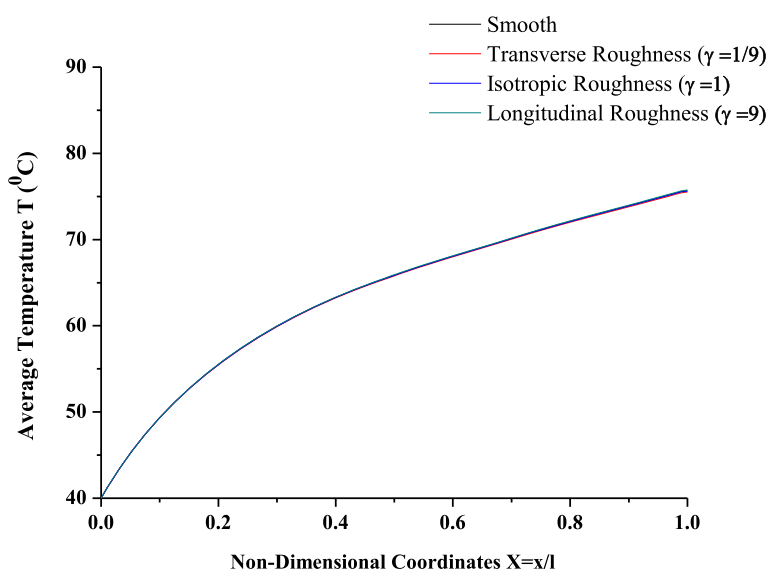

(c)

Fig. 7. Comparison of temperature distribution for different directional orientation of surface roughness and smooth surface at $l=10 \mathrm{~mm}, \quad \eta_{0}=0.01 \mathrm{~Pa} \mathrm{~s}, \quad \rho_{0}=846 \mathrm{~kg} / \mathrm{m}^{3}, \vartheta=0.30, \quad U_{s}=0.05 \mathrm{~m} / \mathrm{s}, \quad \alpha=10 \mathrm{GPa}^{-1}, \quad L_{1}=0.7, \quad h_{1}-h_{2}=100 \mathrm{~nm}, \quad$ and $\Lambda=3$. (a) $h_{2}=1000 \mathrm{~nm}$, (b) $h_{2}=500 \mathrm{~nm}$, and (c) $h_{2}=100 \mathrm{~nm}$.

\subsection{Temperature distribution}

Figure 7 showcases the temperature distribution at different $h_{2}$ for various directional orientations of surface roughness. The temperature distribution with longitudinal and transverse roughness is maximum and minimum, respectively, at all $h_{2}$. This is because the temperature distribution depends on mass flow rate, average film thickness, and difference between pressures of two adjacent nodes (as shown in Eq. (21)). The combined effect of all these parameters causes more viscous shear heating in lubricant, thus, resulting into high temperature under longitudinal orientation compared to transverse. The difference between outlet temperatures of different orientations are negligible for all $h_{2}$. The difference between outlet temperature of transverse and longitudinal roughness increases from $0.0125 \mathrm{C}$ at $1000 \mathrm{~nm}$ to $0.25^{\circ} \mathrm{C}$ at $100 \mathrm{~nm}$. This increase in difference is more at lower value of $h_{2}(100 \mathrm{~nm})$ because of more pressurized shear heating and large difference in total mass flow rate of transverse and longitudinal roughness (Eq. (21)). Due to existence of nonpressure area at smaller value of film thickness, there exists the parabolic nature of temperature distribution at inlet.

\subsection{Flow rates}

Figure 8 shows the flow rates of different roughness orientations at $h_{2}=100 \mathrm{~nm}$ and are compared to flow rates of smooth surfaces. A constant value of the pressureinduced flow rate and couette flow rate exist in $40 \%$ of the contact area from the inlet side for all roughness orientations. This is due to existence of no-pressure zone in this area, which makes the film thickness almost flat (as shown in Fig. 6). The flow rates take the shape of the film thickness, which consists of a flat zone $(0 \leq X \leq 0.4)$, an increasing zone $(0.4 \leq X \leq 0.7)$, a step zone $(0.7 \leq X$ $\leq 0.72)$, and a slightly diverging and converging zone $(0.72 \leq X \leq 1)$. The difference between pressure-induced flow rates of different orientations is very small. Longitudinal roughness has slightly larger value of pressure-induced flow rate compared to other orientations. Transverse roughness has slightly lower value of pressure-induced flow rate compared to other orientations, thus slightly increasing the generated pressure under this orientation compared to others. The difference between Couette flow rates of different orientations is very small. Transverse roughness has slightly higher value of 


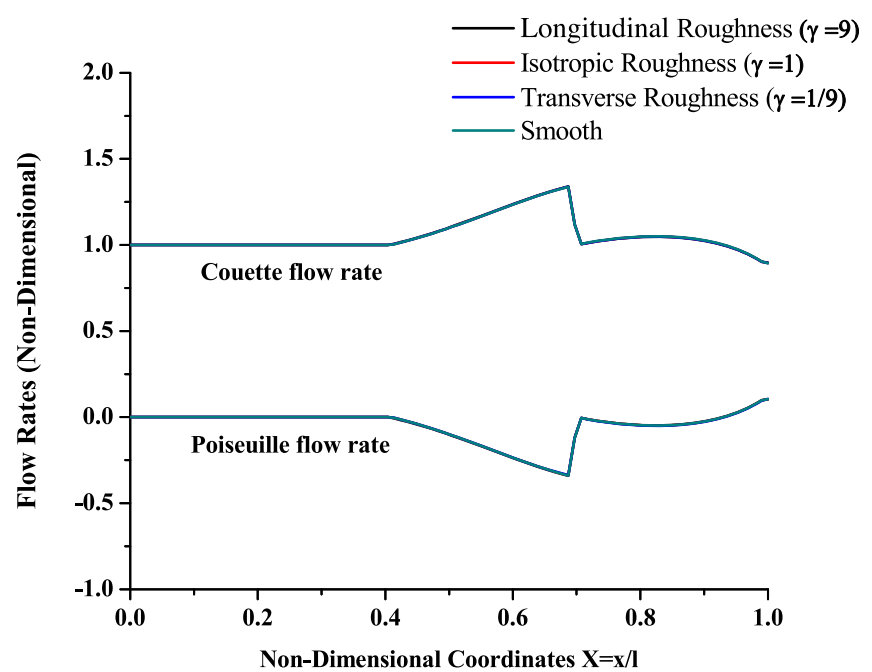

Fig. 8. Comparison between flow rates of different surface roughness orientations at $l=10 \mathrm{~mm}, \eta_{0}=0.01 \mathrm{~Pa} \mathrm{~s}, \rho_{0}=846 \mathrm{~kg} / \mathrm{m}^{3}$, $\vartheta=0.30, U_{s}=0.05 \mathrm{~m} / \mathrm{s}, \alpha=10 \mathrm{GPa}^{-1}, L_{1}=0.7, h_{1}-h_{2}=100 \mathrm{~nm}$, and $\Lambda=3: h_{2}=100 \mathrm{~nm}$.

Couette flow rate compared to other orientations. Longitudinal roughness has slightly lower value of this flow rate compared to others. This is because of the reason that Couette flow rate is proportional to the area of triangle with height $h$ and base $U_{s}$. This area is less in longitudinal roughness compared to transverse, thus resulting in lower value of Couette flow rate in longitudinal roughness compared to others.

\subsection{Effect of hydrodynamic roughness parameter $(\Lambda)$}

Figure 9 shows the effect of $\Lambda$ on various performance parameters of the bearing. Due to large resistance to pressure-induced flow in transverse roughness, the maximum pressure and load capacity at all values of $\Lambda$ is large compared to other orientations (as shown in Figs. 9a and 9b). The difference between load capacities and maximum pressure of different orientation is large at small value of $\Lambda$. This difference decreases with increase in $\Lambda$. At very large value of $\Lambda(\Lambda \geq 6)$, this difference becomes almost negligible and equals to that of smooth surfaces. Total mass flow rate of transverse roughness is large compared to other orientations (as shown in Fig. 9c). This is because of the fact that it is combination of pressure-induced flow rate and Couette flow rate. Pressure-induced flow rate is small and coquette flow rate is large in transverse roughness compared to others. The combined effect of these two flow rates increases the total mass flow rate in transverse roughness. The difference between total mass flow rates of different orientations is large at low values of $\Lambda$. This difference decreases with increase in $\Lambda$ and becomes almost negligible at very high value of $\Lambda$. The maximum temperature of film at outlet is generated in longitudinal roughness for all values of hydrodynamic roughness parameter compared to other orientations (as shown in Fig. 9d). This is because of the fact that fluid under longitudinal roughness undergoes large viscous shear heating. The difference between outlet temperature for all orientations is maximum at lower value of $\Lambda$. This difference decreases with increase in $\Lambda$ and becomes negligibly small at very high values of $\Lambda$.

\subsection{Load capacity}

Figure 10 shows the variation of dimensionless load capacity with respect to film thickness ratios for transverse (Fig. 10a) and longitudinal (Fig. 10b) orientations of surface roughness. It is evident from the graph that the load capacity increases with increase in film thickness. The load capacity for different film thickness increases up to a certain value of $k$. With further increase in $k$, the load capacity decreases and becomes almost constant at very high value of $k$. The difference between load capacity for different $h_{2}$ is large at small value of $k$, which decreases with increase in $k$. This type of behavior in load capacity is witnessed in both type of roughness orientations. With increase in film thickness, the position of maximum load capacity shifts toward low value of film thickness ratios. In case of transverse orientation, the value of $k$ shifts from 5 to 2.1 when the film thickness increases from 100 to $1000 \mathrm{~nm}$, whereas in case of longitudinal roughness, the value of $k$ shifts from 5.2 to 2.3 with increase in film thickness from 100 to $1000 \mathrm{~nm}$.

The load capacity in transverse roughness (as shown in Fig. 10a) is large compared to that in longitudinal (as shown in Fig. 10b) for all values of $h_{2}$. At smaller value of $k$, the sensitivity in load capacity is large in case of transverse orientation compared to longitudinal. Due to this, with increase in $k$, the change in load capacity is small in case of longitudinal roughness compared to transverse for all values of $h_{2}$. This small change tries to slightly flatten the load capacity curve at smaller value of $k$ in case of longitudinal roughness for all $h_{2}$.

Figure 11 showcases the effect of step ratios $\left(L_{1}\right)$ on load capacity for different value of $h_{2}$ under transverse (as shown in Fig. 11a) and longitudinal (as shown in Fig. 11b) orientations. In case of transverse roughness (as shown in Fig. 11a), with increase in film thickness, the value of load capacity increases for all step ratios with some exceptions. At step ratio of around 0.2 , the load capacity at film thickness of $800 \mathrm{~nm}$ are more compared to other $h_{2}$. However, at very small step ratio (around 0.1), the load capacity at film thickness of $600 \mathrm{~nm}$ is large compared to that at other $h_{2}$. With increase in $h_{2}$, the position of maximum load capacity shifts toward large value of step ratios.

In longitudinal roughness (as shown in Fig. 11b), the load capacity increases with increase in film thickness. These values of load capacities are less compared to that under transverse roughness due to increase in the pressure-induced flow (as mentioned in Sect. 3.1), and have some exceptions at lower value of step ratios. At step ratio of around 0.3 and less, the load capacity at film thickness of $600 \mathrm{~nm}$ are large compared to that at other $h_{2}$. At very small step ratio (around 0.2 and less), the load capacity at film thickness of $400 \mathrm{~nm}$ is large compared to that at 800 and $1000 \mathrm{~nm}$. At larger value of step ratio, the load capacity at film thickness of $800 \mathrm{~nm}$ is 


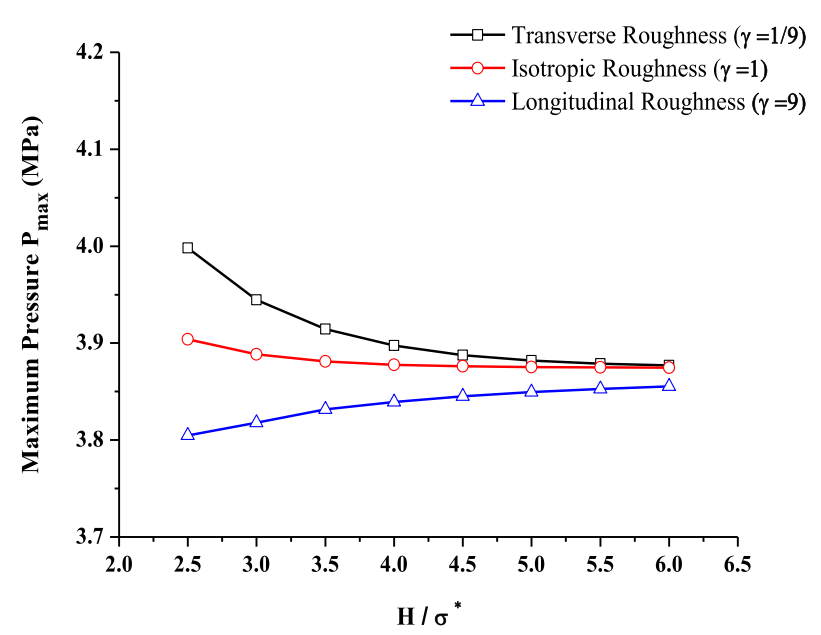

(a)

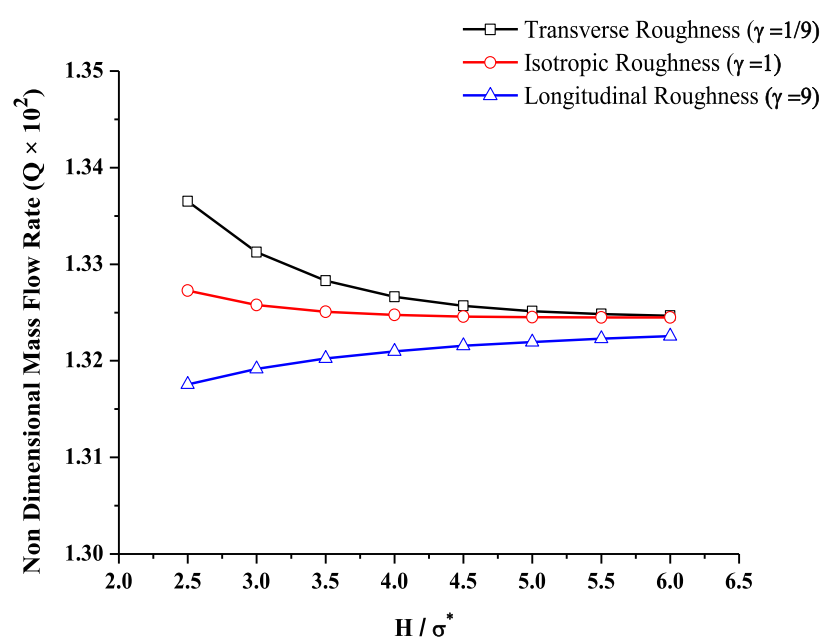

(c)

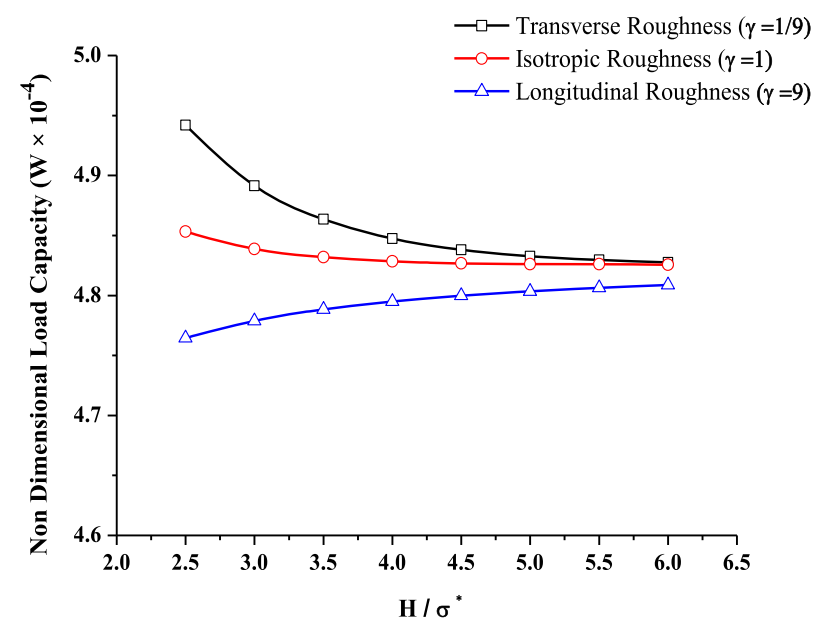

(b)

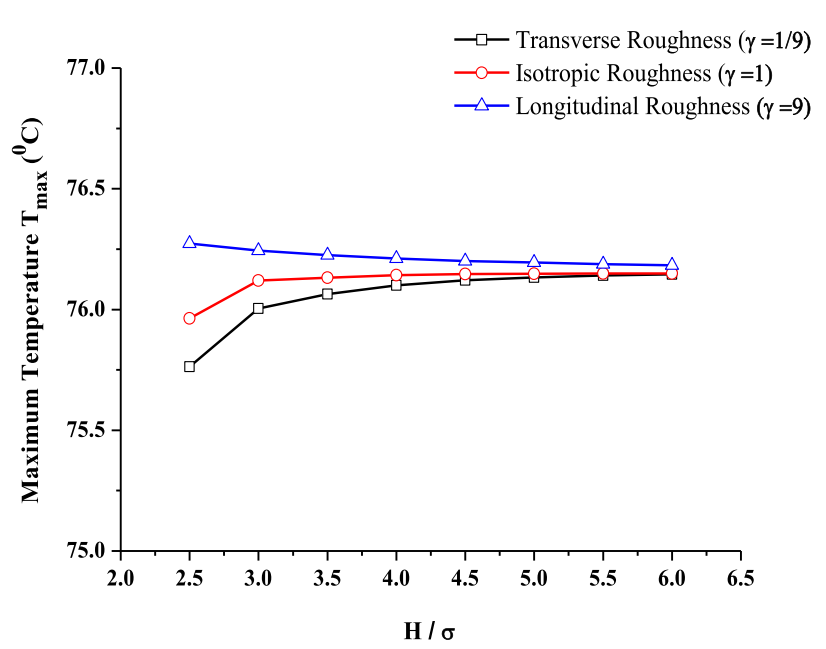

(d)

Fig. 9. Effect of hydrodynamic roughness parameter on (a) maximum pressure, (b) load capacity, (c) mass flow rate, and (d) maximum temperature at $l=10 \mathrm{~mm}, \eta_{0}=0.01 \mathrm{~Pa} \mathrm{~s}, \rho_{0}=846 \mathrm{~kg} / \mathrm{m}^{3}, \vartheta=0.30, U_{s}=0.05 \mathrm{~m} / \mathrm{s}, \alpha=10 \mathrm{GPa}^{-1}, L_{1}=0.7, h_{1}-h_{2}=$ $100 \mathrm{~nm}$, and $h_{2}=100 \mathrm{~nm}$.

large compared to that at $1000 \mathrm{~nm}$. The position of maximum load capacity follows the trend of transverse roughness.

\subsection{Coefficient of friction}

Figure 12 shows the variation of dimensionless frictional coefficient with respect to $k$ at different minimum film thickness for both transverse (as shown in Fig. 12a) and longitudinal (as shown in Fig. 12b) roughness patterns. It is evident from the graph that at lower value of $k$, the frictional coefficient is maximum for smaller film thickness. This is due to large decrement in load capacity at smaller film thickness and low film thickness ratios (as shown in Figs. 10a and 10b). With increasing film thickness, the value of frictional coefficient decreases. At higher value of film thickness ratio $(k>8)$, the frictional coefficient becomes more for thick film $(1000 \mathrm{~nm})$ compared to thin film $(100 \mathrm{~nm})$. The difference between frictional coefficient for various film thickness becomes small at higher value of $k$. This trend of frictional coefficient variation with respect to film thickness ratios is same for both transverse and longitudinal roughness. The position of minimum frictional coefficient shifts toward lower value of $k$ with increasing film thickness. In case of transverse orientation, the value of $k$ shifts from 5.5 to 1.8 with increase in $h_{2}$ from 100 to $1000 \mathrm{~nm}$ for minimum frictional coefficient, whereas the value of $k$ shifts from 6 to 2 in case of longitudinal orientation with increase in film thickness from 100 to $1000 \mathrm{~nm}$ for minimum frictional coefficient. The frictional coefficient in longitudinal roughness is slightly larger in comparison to transverse roughness due to decrement in load capacity under longitudinal roughness (as shown in Fig. 10).

Figure 13 showcases the effect of step ratios on frictional coefficient. With increasing $h_{2}$, the frictional coefficient decreases up to certain film thickness and then increases for transverse (as shown in Fig. 13a) and longitudinal 


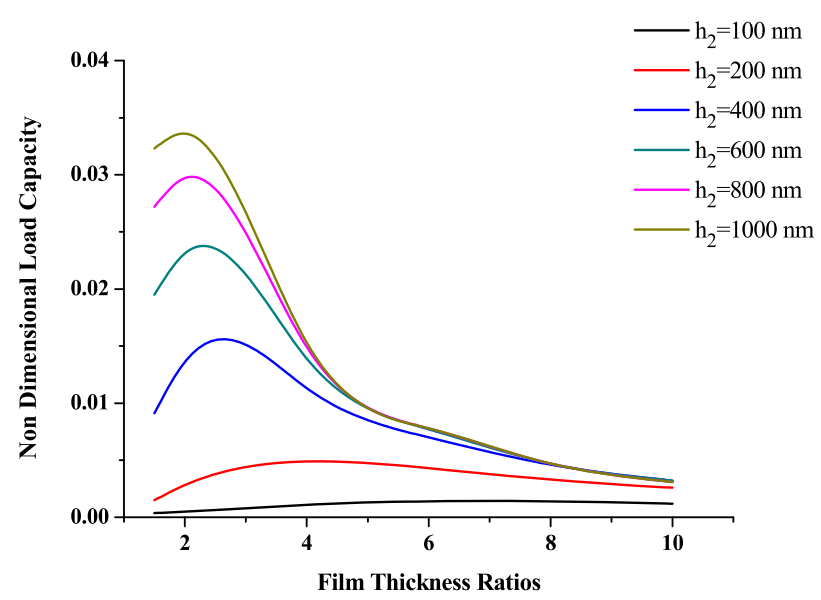

(a)

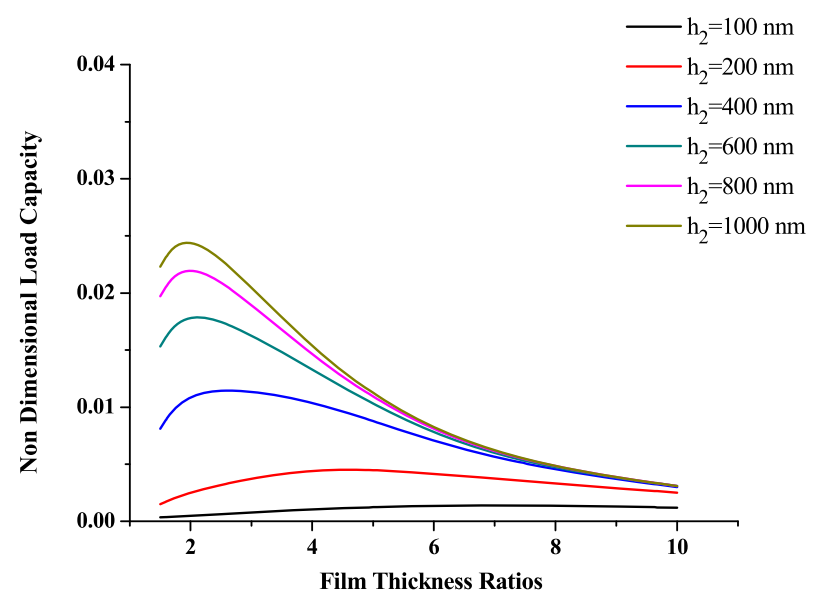

(b)

Fig. 10. Comparison between nondimensional load capacity with variable film thickness ratio for (a) transverse roughness orientation and (b) longitudinal roughness orientation for different values of $h_{2}$ at $l=10 \mathrm{~mm}, \eta_{0}=0.01 \mathrm{~Pa} \mathrm{~s}, \rho_{0}=846 \mathrm{~kg} / \mathrm{m}^{3}, \vartheta=0.30, U_{s}=0.05 \mathrm{~m} / \mathrm{s}$, $\alpha=10 \mathrm{GPa}^{-1}, L_{1}=0.7$, and $\Lambda=3$.

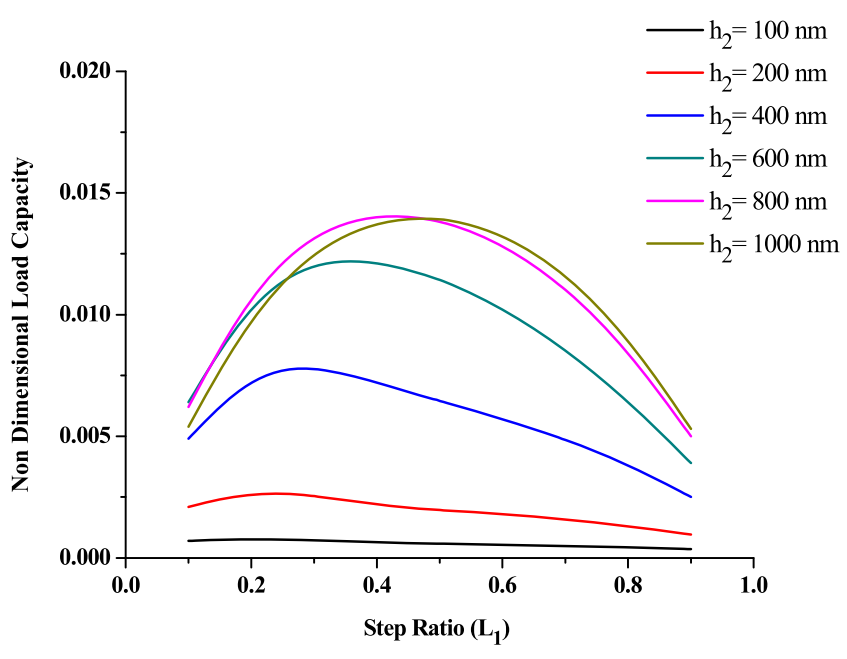

(a)

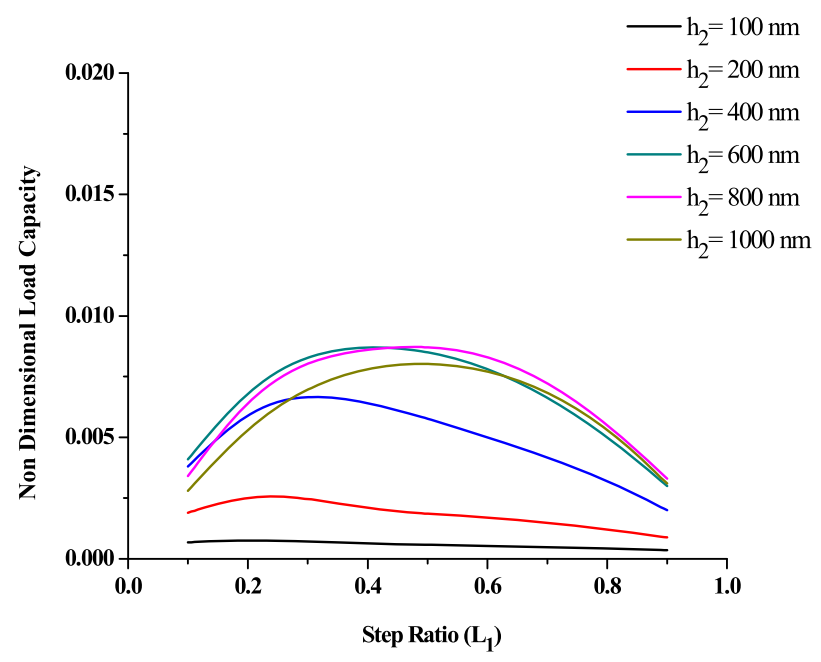

(b)

Fig. 11. Comparison between nondimensional load capacity with variable step ratio for (a) transverse roughness orientation and (b) longitudinal roughness orientation for different values of $h_{2}$ at $l=10 \mathrm{~mm}, \eta_{0}=0.01 \mathrm{~Pa} \mathrm{~s}, \rho_{0}=846 \mathrm{~kg} / \mathrm{m}^{3}, \vartheta=0.30, U_{s}=0.05 \mathrm{~m} / \mathrm{s}$, $\alpha=10 \mathrm{GPa}^{-1}, h_{1}-h_{2}=100 \mathrm{~nm}$, and $\Lambda=3$.

roughness (as shown in Fig. 13b). This is due to change in load capacity at various step ratios (as shown in Fig. 11). At lower value of $L_{1}\left(L_{1}<0.3\right)$, the difference between frictional coefficient for different $h_{2}$ is large. The minimum value of frictional coefficient exists at lower step ratio (at around 0.3 ). At very low value of step ratio, the coefficient of friction for 100 and $200 \mathrm{~nm}$ is small compared to other film thicknesses for both orientations. This is due to reduction in frictional force at this step ratio. With further increment in step ratio, the difference between frictional coefficient of different $h_{2}$ decreases. There exists an exception at 100 and $200 \mathrm{~nm}$ where this difference remains almost constant. The value of frictional coefficient becomes almost constant under both orientations for all other $h_{2}$ with increasing step ratio $\left(0.3 \leq L_{1} \leq 0.7\right)$. But, at 100 and $200 \mathrm{~nm}$, the value of frictional coefficient constantly increases with increase in step ratio. At very high value of step $\operatorname{ratio}\left(L_{1}>0.7\right)$, the difference between frictional coefficients of various $h_{2}$ further decreases. The value of coefficient of friction at high step ratio increases for all $h_{2}$. This trend of frictional coefficient under both orientations is due to combined effect of load capacity (as shown in Fig. 11) and frictional force on moving surface. The value of frictional coefficient under transverse roughness is small 


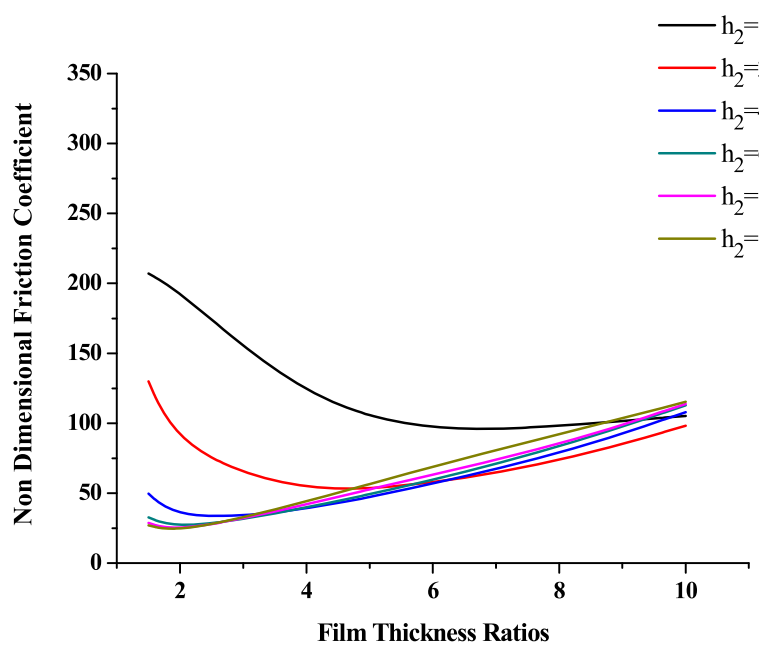

(a)

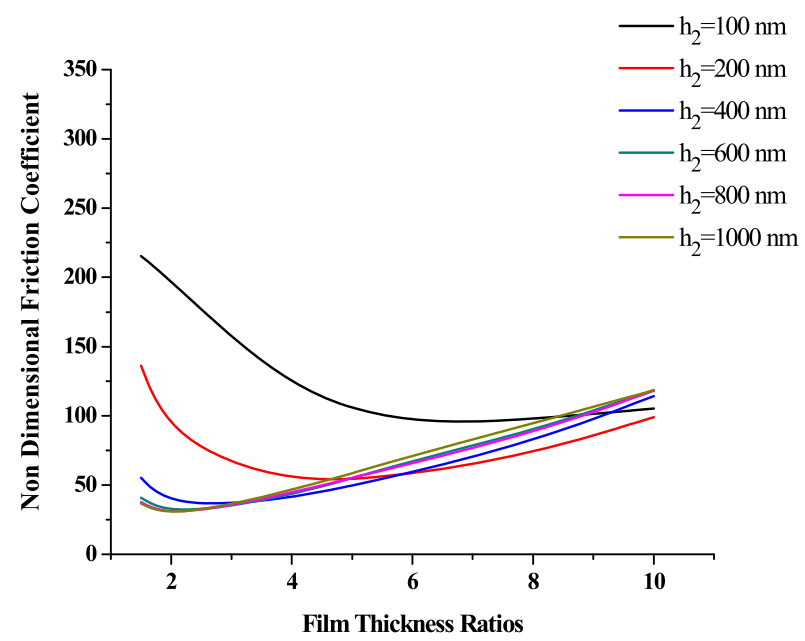

(b)

Fig. 12. Comparison between nondimensional frictional coefficient with variable film thickness ratio for (a) transverse roughness orientation and (b) longitudinal roughness orientation for different values of $h_{2}$ at $l=10 \mathrm{~mm}, \eta_{0}=0.01 \mathrm{~Pa} \mathrm{~s}, \rho_{0}=846 \mathrm{~kg} / \mathrm{m}^{3}, \vartheta=0.30$, $U_{s}=0.05 \mathrm{~m} / \mathrm{s}, \alpha=10 \mathrm{GPa}^{-1}, L_{1}=0.7$, and $\Lambda=3$.

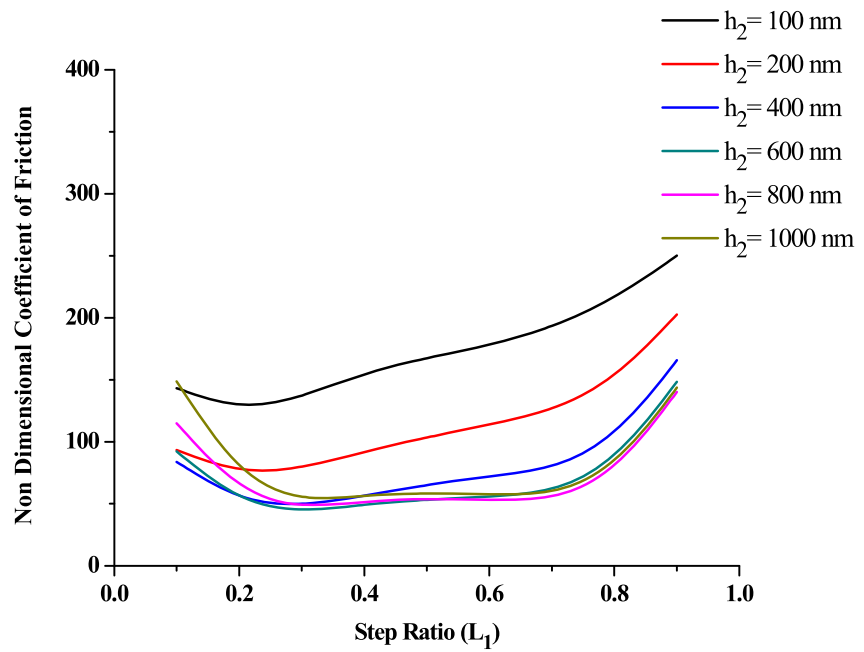

(a)

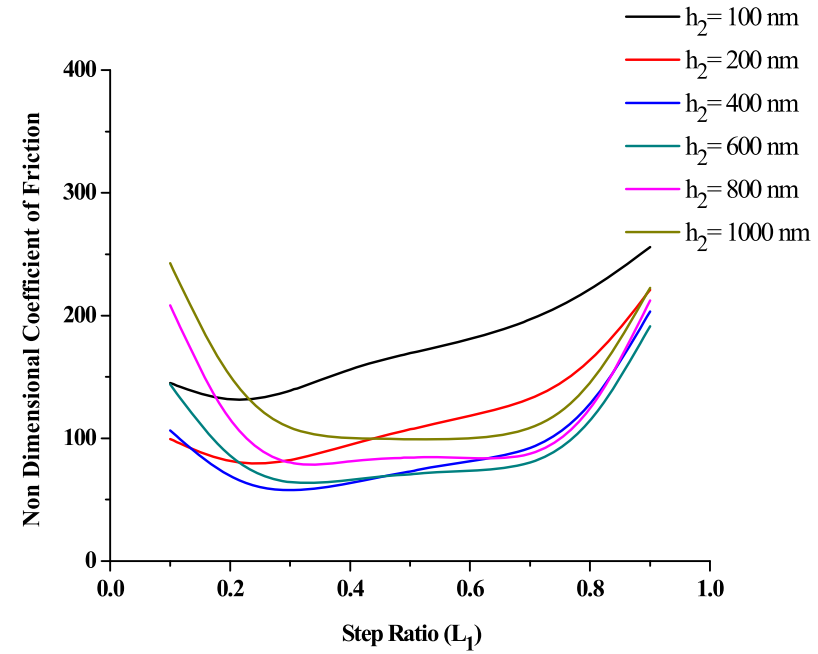

(b)

Fig. 13. Comparison between nondimensional frictional coefficient with variable step ratio for (a) transverse roughness orientation and (b) longitudinal roughness orientation for different values of $h_{2}$ at $l=10 \mathrm{~mm}, \eta_{0}=0.01 \mathrm{Pas}, \rho_{0}=846 \mathrm{~kg} / \mathrm{m}^{3}, \vartheta=0.30, U_{s}=0.05 \mathrm{~m} / \mathrm{s}$, $\alpha=10 \mathrm{GPa}^{-1}, h_{1}-h_{2}=100 \mathrm{~nm}$, and $\Lambda=3$.

compared to that under longitudinal roughness. This is due to reduction in load capacity under longitudinal roughness compared to transverse roughness (as shown in Fig. 11).

\subsection{Effect of material parameters}

Figure 14 compares the effect of different material parameters on pressure distribution for both transverse (Fig. 14a) and longitudinal (Fig. 14b) orientations. It is evident from the graph that materials with low modulus of elasticity undergo more surface deformation even at small pressure compared to material with high elastic modulus.
With increase in elastic modulus, the area under no pressure zone decreases. At low elastic modulus, two prominent pressure peaks get generated. In between these two pressure peaks, there exists a small area where pressure is very low (almost zero). This low pressure area decreases with increase in elastic modulus. Also, the generated pressure under longitudinal orientation of roughness for all elastic modulus is less compared to transverse orientation of roughness due to reason mentioned in Section 3.2. The no-pressure zone under longitudinal orientation is slightly small compared to that under transverse orientation due to less surface deformation and more pressure-induced flow rate under longitudinal orientation. 


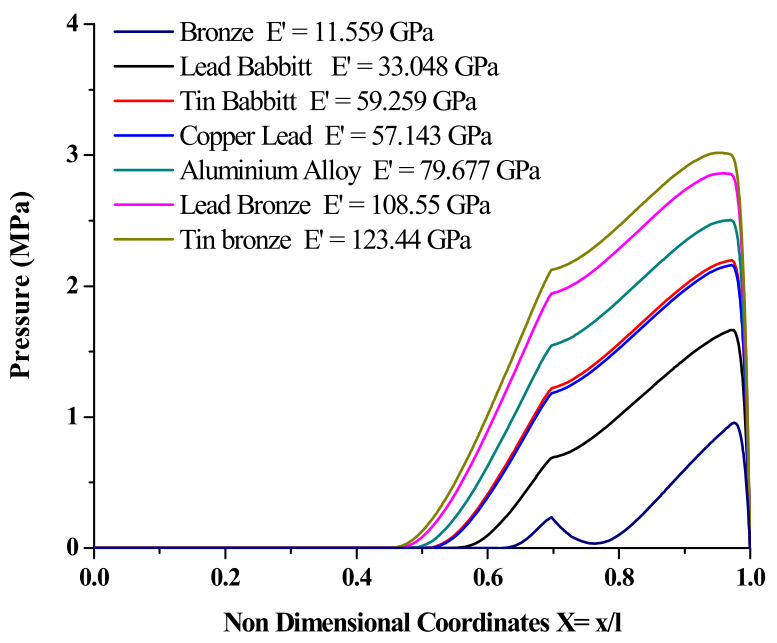

(a)

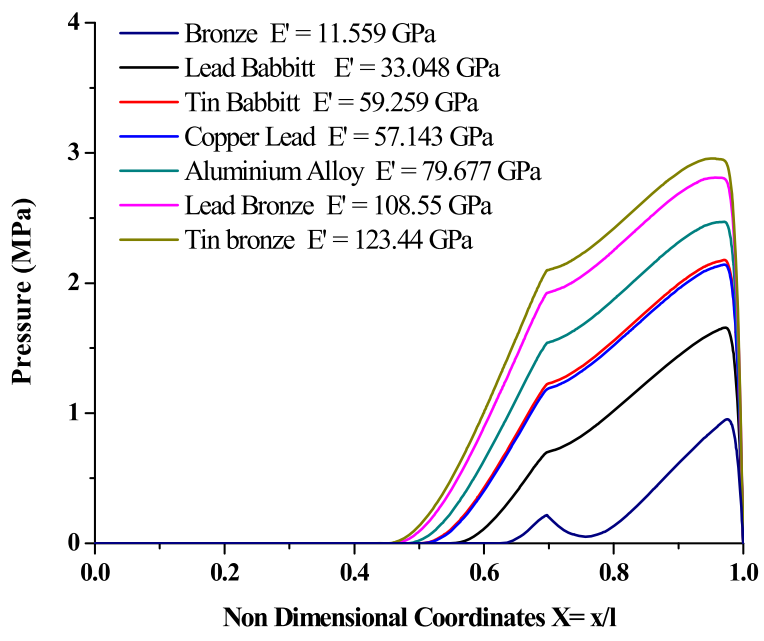

(b)

Fig. 14. Comparison of pressure distribution for (a) transverse and (b) longitudinal roughness orientation with different material parameters at $l=10 \mathrm{~mm}, \eta_{0}=0.01 \mathrm{~Pa} \mathrm{~s}, \rho_{0}=846 \mathrm{~kg} / \mathrm{m}^{3}, U_{s}=0.05 \mathrm{~m} / \mathrm{s}, \alpha=10 \mathrm{GPa}^{-1}, L_{1}=0.7, h_{1}-h_{2}=100 \mathrm{~nm}, h_{2}=100 \mathrm{~nm}$, $T_{0}=40^{\circ} \mathrm{C}$, and $\Lambda=3$.

\section{Conclusion}

This study investigates the effect of stochastic roughness on performance parameters of Rayleigh step bearing under thin film lubrication. The following conclusions have been drawn from the generated results:

- The nature and directional orientation of the stochastic roughness play a significant role in pressure generation. Among all orientations, transverse roughness generates more pressure compared to others at various film thicknesses. The difference between generated pressures under different types of orientations goes on decreasing with decrease in film thickness.

- The temperature distribution under longitudinal orientation is slightly more compared to other orientations at different film thicknesses. This is due to slightly increased shear heating under this orientation resulting from combined effect of mass flow rate, average film thickness, and difference between pressures of two adjacent nodes. The temperature distribution between different roughness cases is almost identical, with insignificant difference for all values of film thickness.

- Maximum pressure, mass flow rate, and load capacity is large in transverse roughness compared to other for all values of hydrodynamic roughness parameters. With increasing hydrodynamic roughness parameter, these values decrease and become almost constant and equal to that of smooth surface. At very high hydrodynamic roughness parameters, these values become almost equal to that of smooth surface.

- Maximum temperature at outlet is large in longitudinal roughness compared to others for all hydrodynamic roughness parameters. At very high hydrodynamic roughness parameter, the maximum temperature decreases and becomes almost equal to that under smooth surface.
- Load capacity under transverse roughness at all values of film thickness ratios and step ratios for different film thickness is large in comparison to longitudinal roughness. There exists large sensitivity in load capacity for transverse roughness compared to longitudinal roughness at higher film thickness at different film thickness ratios. At very high film thickness ratios, load capacity for different film thickness becomes almost constant for both (longitudinal and transverse) orientations. With increasing film thickness, the value of maximum load capacity shifts toward low film thickness ratios and high step ratios.

- Coefficient of friction in longitudinal roughness is large compared to transverse at all values of film thickness ratios and step ratios for different film thickness. At high film thickness ratios and step ratios, the difference between coefficient of friction for different film thickness becomes less compared to that at low film thickness ratios and step ratios. With increasing film thickness, the position for minimum coefficient of friction shifts toward low value of film thickness ratios and high value of step ratios.

- Materials with low elastic modulus undergo more surface deformation even at small pressure compared to material with high elastic modulus, and two prominent pressure peaks get generated. With increase in elastic modulus, the area under no pressure zone decreases.

The current investigation suggests that directional orientation of surface roughness influences the performance parameters of the bearing greatly under thin film lubrication. However, a clear understanding of phenomenon related to rough thin film lubrication needs further investigation. The main reason is that the present analysis is based on the assumption of same roughness orientations and same standard deviation of roughness heights on interacting surfaces. However, these surfaces may have same roughness orientations but different standard 
deviations of roughness height, this condition needs further investigation to study the effect of such roughness parameters on bearing performance in more detail.

\section{Nomenclature}

$h \quad$ Film thickness $(\mathrm{m})$

$H \quad$ Nondimensional film thickness $H={ }^{h} / h_{2}$

$p \quad$ Fluid film pressure $(\mathrm{Pa})$

$P \quad$ Nondimensional pressure of the fluid film $P=p h_{2}^{2} / 6 U_{s} l \eta_{0}$

$l \quad$ Width of the pad $(\mathrm{m})$

$X \quad$ Nondimensional width of the pad $X={ }^{x} / l$

$\eta_{0} \quad$ Viscosity at $p=0(\mathrm{~Pa} \mathrm{~s})$

$\rho_{0} \quad$ Density at $p=0\left(\mathrm{~kg} / \mathrm{m}^{3}\right)$

$\eta_{*} \quad$ Lubricant's viscosity (Pa s)

$\eta^{*} \quad$ Nondimensional viscosity $\eta^{*}=\eta / \eta_{0}$

$\rho_{*} \quad$ Lubricant's density $\left(\mathrm{kg} / \mathrm{m}^{3}\right)$

$\rho^{*} \quad$ Nondimensional density $\rho^{*}=\rho / \rho_{0}$

$h_{1} \quad$ Film thickness at inlet $(\mathrm{m})$

$h_{2} \quad$ Film thickness at outlet $(\mathrm{m})$

$U_{s} \quad$ Moving surface sliding velocity $(\mathrm{m} / \mathrm{s})$

$h_{\text {avg }} \quad$ Average film thickness $h_{\text {avg }}=h+\delta_{1}+\delta_{2}(\mathrm{~m})$

$H_{\text {avg }} \quad$ Nondimensional average film thickness

$\delta_{1}, \delta_{2}$ Roughness amplitudes of surfaces (m)

$\delta \quad$ Combined surface roughness amplitude $\delta=\delta_{1}+$ $\delta_{2}(\mathrm{~m})$

$\sigma_{1}, \sigma_{2}$ Standard deviation of surface roughness of roughness amplitudes (m)

$\sigma \quad$ Standard deviation of combined surface roughness amplitude $\sigma=\sqrt{\sigma_{1}^{2}+\sigma_{2}^{2}}(\mathrm{~m})$

$\sigma^{*} \quad$ Nondimensional standard deviation of combined surface roughness amplitude

$\phi_{x} \quad$ Pressure flow factor

$d \quad$ Equivalent elastic deformation of the surfaces $(\mathrm{m})$

$d^{*} \quad$ Nondimensional equivalent elastic deformation of the surfaces

$E_{1} \quad$ Elastic modulus of stationary pad (GPa)

$E_{2} \quad$ Elastic modulus of moving surface (GPa)

$E^{\prime} \quad$ Equivalent elastic modulus of surfaces (GPa)

$\vartheta_{1} \quad$ Poisson's ratio of stationary pad

$\vartheta_{2} \quad$ Poisson's ratio of moving surface

$k \quad$ Film thickness ratio $k=h_{1} / h_{2}$

$\beta \quad$ Coefficient of lubricant thermal expansivity $\left({ }^{\circ} \mathrm{C}^{-1}\right)$

$\alpha \quad$ Pressure viscosity coefficient $\left(\mathrm{Pa}^{-1}\right)$

$C_{p} \quad$ Specific heat of the lubricant $\left(\mathrm{J} \mathrm{Kg}^{-1}{ }^{\circ} \mathrm{C}^{-1}\right)$

$q \quad$ Mass flow rate $\left(\mathrm{Kg} \mathrm{m}^{-1} \mathrm{~s}^{-1}\right)$

$Q \quad$ Nondimensional mass flow rate $Q=\frac{q}{\rho_{0} h_{2}}$

$q_{c} \quad$ Couette mass flow rate $\left(\mathrm{Kg} \mathrm{m}^{-1} \mathrm{~s}^{-1}\right) \frac{q_{0} U_{s} h_{2}}{\rho_{c}}$

$Q_{c} \quad$ NonDimensional Couette mass flow rate $Q_{c}=\frac{q_{c}}{\rho_{0} U_{s} h_{2}}$

$q_{p} \quad$ Poiseuille mass flow rate $\left(\mathrm{Kg} \mathrm{m}^{-1} \mathrm{~s}^{-1}\right)$

$Q_{p} \quad$ Nondimensional Poiseuille mass flow rate $Q_{p}=\frac{q_{p}}{\rho_{0} U_{s} h_{2}}$

$w_{*} \quad$ Load capacity $(\mathrm{N} / \mathrm{m})$

$w^{*} \quad$ Nondimensional load capacity $w^{*}=\frac{w h_{2}{ }^{2}}{6 \eta_{0} U_{s} l^{2}}$

$f \quad$ Frictional force $(\mathrm{N})$

$F \quad$ Nondimensional frictional force

$F^{*} \quad$ Nondimensional coefficient of friction

\section{References}

[1] T.E. Carl, An experimental investigation of a cylindrical journal bearing under constant and sinusoidal loading, Proc. Inst. Mech. Eng. 178 (1963) 100-119. doi: 10.1243/PIME

[2] E.W. Hemingway, The measurement of film thickness in thrust bearings and the deflected shape of "parallel" surface thrust pads, Proc. Inst. Mech. Eng. 180 (1965) 1025-1034

[3] S. Kawabata, S. Iwanami, T. Hotta, F. Itoigawa, T. Nakamura, Hydrodynamic lubrication effects of multiple circular bump pattern for a thrust sliding bearing of a scroll compressor, Tribology 7 (2012) 13-23. doi: 10.2474/trol.7.13

[4] A. Bennett, C. Ettles, A self-acting parallel surface thrust bearing, Proc. Inst. Mech. Eng. 182 (1967) 139-146

[5] C.L. Robinson, A. Cameron, Studies in hydrodynamic thrust bearings. II. Comparison of calculated and measured performance of tilting pads by means of interferometry, Philos. Trans. R. Soc. Lond. A 278 (1975) 367-384. doi: 10.1098/rsta.1948.0007

[6] A. Cameron, C.L. Robinson, Studies in hydrodynamic thrust bearings. I. Theory considering thermal and elastic destortions, Philos. Trans. R. Soc. Lond. A 278 (1975) 351-366. doi: 10.1098 /rsta.1948.0007

[7] C.M. Ettles, Size effects in tilting pad thrust bearings, Wear 59 (1980) 231-245

[8] A. Cameron, The Viscosity Wedge, ASLE Trans. 1 (1958) 248-253. doi: 10.1080/05698195808972337

[9] J. Lundberg, Influence of surface roughness on normalsliding lubrication, Tribol. Int. 28 (1995) 317-322. doi: 10.1016/0301-679X(94)00003-9

[10] V. Bakolas, Analysis of rough line contacts operating under mixed elastohydrodynamic lubrication conditions, Lubr. Sci. 16 (2004) 153-168. doi: 10.1002/ls.3010160206

[11] S.T. Tzeng, E. Sabiel, Surface roughness effect on slider lubrication, ASLE Trans. 10 (1967) 334-348

[12] H. Christensen, Stochastic models for hydrodynamic lubrication of rough surfaces, Proc. Inst. Mech. Eng. 184 (1970) 1013-1026

[13] H. Christensen, K. Tonder, The hydrodynamic lubrication of rough bearing surfaces of finite width, J. Tribol. 93 (1971) 324-329

[14] K. Tonder, H. Christensen, The hydrodynamic lubrication of rough journal bearings, J. Tribol. 95 (1973) 166-172

[15] L.S.H. Chow, H.S. Cheng, Influence of Surface Roughness and Waviness on Film Thickness and Pressure Distribution in Elastohydrodynamic Contacts, NASA Contractor Report CR-2670 (1967)

[16] H.S. Cheng, L.S.H. Chow, The effect of surface roughness on the average film thickness between lubricated rollers, J. Tribol. 98 (1976) 117-124

[17] N. Patir, H.S. Cheng, Average flow model for determining effects of 3-dimensional roughness on partial hydrodynamic lubrication, J. Lubr. Technol. 100 (1978) 12-17

[18] N. Patir, H.S. Cheng, Application of average flow model to lubrication between rough sliding surfaces, J. Lubr. Technol. 101 (1979) 220-230

[19] H.S. Cheng, N. Patir, Effect of surface roughness orientation on the central film thickness in EHD contacts, Proc. Soc. Photo-Opt. Instrum. Eng. (1979) 15-21

[20] F. Guo, X. Jia, S. Suo, R.F. Salant, Y. Wang, A mixed lubrication model of a rotary lip seal using flow factors, Tribol. Int. 57 (2013) 195-201 
[21] Q. Wen, Y. Liu, W. Huang, S. Suo, Y. Wang, The effect of surface roughness on thermal-elasto-hydrodynamic model of contact mechanical seals, Sci. China Phys. Mech. Astron. 56 (2013) 1920-1929

[22] X.Y. Zhao, Y. Liu, W.F. Huang, X.F. Liu, Y.M. Wang, Mechanism of second stage mechanical seal in hydrostatic seal system of nuclear coolant pumps, Tribology 4 (2014) 459-467

[23] H. Khan, P. Sinha, Thermal elastohydrodynamic lubrication of line contact rough surfaces using flow factor method, Contemp. Eng. Sci. 3 (2010) 113-138

[24] H. Khan, P. Sinha, Effect of shear flow factor on thermal elastohydrodynamic lubrication of infinite line contact rough surfaces, Proc. Natl. Acad. Sci. A 80 (2010) 327-346

[25] H. Khan, Thermal elastohydrodynamic lubrication of infinite line contact rough surfaces considering pressure and shear flow factors, Int. J. Surf. Sci. Eng. 7 (2013) 217-249

[26] H. Khan, P. Sinha, Effect of inter-asperity cavitation on thermal elastohydrodynamic lubrication of infinite line contact rough surfaces, Int. J. Surf. Sci. Eng. 5 (2011) $2015-225$

[27] M. Qui, B. Raeymaekers, The load-carrying capacity and friction coefficient of incompressible textured parallel slider bearings with surface roughness inside the textured features, Proc. Mech. Eng. J 229 (2015) 547-556

[28] Y. Wang, Y. Liu, Z. Wang, Y. Wang, Surface roughness characteristics effects on fluid load capability of tilting pad thrust bearings with water lubrication, Friction 5 (2017) 392-401

[29] R.A. Burton, Effect of two-dimensional, sinusoidal roughness on the load support characteristics of a lubricant film, J. Basic Eng. D 85 (1963) 258-262

[30] M.G. Davies, The generation of pressure between rough, fluid lubricated, moving, deformable surfaces, Lubr. Eng. 19 (1963) 246.

[31] A. Felix Quinonenz, G.E. Morales-Espejel, Surface roughness effects in hydrodynamic bearings, Tribol. Int. 98 (2016) $212-219$
[32] R. Kumar, Md. S. Azam, S.K. Ghosh, H. Khan, Effect of surface roughness and deformation on Rayleigh step bearing under thin film lubrication, Ind. Lubr. Tribol. 69 (2017) 1016-1032

[33] P.R. Goglia, T.F. Conry, C. Cusano, The effects of surface irregularities on the elastohydrodynamic lubrication of sliding line contacts. 1. Single irregularities, J. Tribol. 106 (1984) 104-112

[34] P.R. Goglia, T.F. Conry, C. Cusano, The effects of surface irregularities on the elastohydrodynamic lubrication of sliding line contacts. 2. Wavy surfaces, J. Tribol. 106 (1984) 113-119

[35] C.C. Kweh, H.P. Evans, R.W. Sindle, Micro-elastohydrodynamic lubrication of an elliptical contact with transverse and tree-dimensional sinusoidal roughness, J. Tribol. 111 (1989) $577-584$

[36] H. Khan, P. Sinha, A. Saxena, A simple algorithm for thermo-elasto-hydrodynamic lubrication problems, Int. J. Res. Rev. Appl. Sci. 1 (2009) 265-279

[37] D.S. Wang, J.F. Lin, Effect of surface roughness on elastohydrodynamic lubrication of line contacts, Tribol. Int. 24 (1991) 51-62

[38] H. Khan, A numerical study of thermo elastohydrodynamic lubrication of infinite line contact rough surfaces, $\mathrm{PhD}$ thesis, 2010

[39] G. Higginson, D. Dowson, Elastohydrodynamic Lubrication, Pergamon Press, Oxford, 1977

[40] C. Roelands, J. Vlugter, H. Watermann, The viscositytemperature-pressure relationship of lubricating oils and its correlation with chemical constitution, ASME J. Basic Eng. 85 (1963) 601-610

[41] P. Huang, Numerical Calculation of Lubrication, 1st edn, John Wiley \& Sons, Inc., Guangzhou, 2013, pp. 117-124

[42] D. Zhu, On some aspects in numerical solution of thin-film and mixed EHL, J. Eng. Tribol. 221 (2007) 561-579. doi: 10.1243/13506501JET259

[43] K. Yagi, J. Sugimura, Elastohydrodynamic simulation of Rayleigh step bearings in thin film hydrodynamic lubrication, Tribol. Int. $64 \quad(2013)$ 214. doi: 10.1016/j.tri boint.2013.04.005

Cite this article as: R. Kumar, M.S. Azam, S.K. Ghosh, H. Khan, Performance evaluation of rough thrust pad bearing under thermo-elastohydrodynamic lubrication using an improved iterative method, Mechanics \& Industry 20, 110 (2019) 